\title{
Vegetación de bordes en un Bosque Mesófilo de Montaña del Occidente de México
}

\author{
Ana Luisa Santiago-Pérez ${ }^{1,4}$, Enrique José Jardel-Peláez², Ramón Cuevas-Guzmán² \\ y Francisco Martín Huerta-Martínez ${ }^{3}$ \\ 'Departamento de Producción Forestal, Universidad de Guadalajara-CUCBA. \\ Km. 15.5 Carretera Guadalajara - Nogales, Las Agujas, Zapopan, 45020, Jalisco, México. \\ 2Instituto Manantlán de Ecología y Conservación de la Biodiversidad, Universidad de Guadalajara-CUCSUR. \\ ${ }^{3}$ Departamento de Ecología, Universidad de Guadalajara-CUCBA. \\ ${ }^{4}$ Autor para la correspondencia. Correo-e: ana.santiago@cucba.udg.mx
}

\begin{abstract}
Resumen: La composición, diversidad, estructura y microclima fueron comparadas en dos tipos de bordes de bosque mesófilo de montaña (BMM) en la Sierra de Manantlán (Jalisco, México), uno con borde suave (B-B, contiguo a bosque de pino-encino) y otro con borde abrupto (B-M, contiguo a matorral secundario). Se establecieron 9 parcelas permanentes ( $\mathrm{n}=5$ en B-B y $4=$ en B-M) perpendiculares al borde. El BMM presentó mayor riqueza (126 especies, de estas 52 exclusivas al interior) que el bosque de pino (84) y los matorrales (71). La riqueza y diversidad fueron similares en B-B, pero el reemplazo de especies fue mayor en B-M. La densidad, diámetros, área basal y cobertura del dosel fueron mayores en B-B. La variación en las condiciones del microclima y suelos fueron mayores en los B-M. Las especies arbóreas de BMM (alta densidad de juveniles y plántulas $<5 \mathrm{~cm}$ de diámetro) están colonizando el sotobosque del bosque de pino adyacente, mientras que en los matorrales su establecimiento parece estar limitado por la competencia con los arbustos y hierbas, además de las condiciones del microclima. Los resultados obtenidos resaltan la importancia de considerar el contraste entre bordes en la conservación y restauración del BMM en paisajes forestales.
\end{abstract}

Palabras clave: tipos de bordes, bosques subtropicales de montaña, mosaico forestal, Sierra de Manantlán.

Abstract: Species composition, diversity, structure and microclimate were compared in two edge type of montane cloud forest (FF, edges with pine forest and FS, secondary shrubland) within a forest-edge-exterior gradient at Sierra de Manantlán Biosphere Reserve (Jalisco, Mexico). The cloud forest presented higher richness (126 species, 52 interior habitat specific) than pine forests (84) and shrublands (71). Richness and diversity were similar in FF, but species replacement was higher in FS. Density, diameter structure, basal area and canopy cover were major in FF than in FS. Microclimatic and soil condition, was gradual in FF edges and abrupt in FS edges. Tree species of the cloud forests (high density of seedlings and saplings $<5 \mathrm{~cm}$ diameter breast height) are colonizing the understory of adjacent pine forests, whereas in shrublands their establishment appears to be limited by competition with shrubs and herbaceous species and microclimatic conditions. Our results highlight the importance of considering edge type contrast in conservation and restoration of cloud forest in forest landscapes.

Key words: edge type, subtropical montane forests, forest mosaic, Sierra de Manantlán.

$\mathbf{L}^{2}$ a pérdida de hábitat ocasionada por la deforestación se encuentra entre las principales causas de extinción de especies en ecosistemas forestales (Lindenmayer y Franklin, 2002; Shvidenko et al., 2005). Una de las consecuencias de la deforestación es la fragmentación de los bosques y el aumento de la proporción de bordes con hábitats transformados adyacentes (Lindenmayer y Fischer, 2006). En estos bordes se manifiestan influencias bióticas y abióticas en la composición de especies, estructura y funcionamiento de los ecosistemas que contrastan con las condiciones del interior de los parches de hábitat original remanente (Harper et al., 2005).

Aunque algunos de los primeros estudios sobre bordes resaltaban un efecto favorable para ciertas especies, como aves rapaces o animales de caza (Leopold, 1933), se han documentado varias consecuencias negativas del efecto de borde como el aumento del riesgo de extinción local de especies de plantas y animales típicas del interior del bosque 
(Murcia, 1995; Fahrig, 2003; Broadbent et al., 2008). Los estudios sobre la fragmentación y el efecto de borde entre hábitats naturales y transformados, han ocupado un lugar central en el campo de la conservación biológica (Harper et al., 2005), ya que el entendimiento de sus consecuencias es relevante para diseñar prácticas adecuadas de gestión de áreas protegidas, conservación de biodiversidad en bosques manejados con fines productivos y restauración de hábitat (Lindenmayer y Franklin, 2002).

En México el bosque mesófilo de montaña (BMM) ha recibido particular atención en los estudios sobre efectos de borde, ya que se trata de un tipo de hábitat amenazado, cuya superficie se ha reducido y fragmentado significativamente (Challenger, 1998). El BMM es una prioridad para la conservación (Jardel-Peláez et al., 1993; Challenger, 1998; Williams-Linera et al., 2002) por sus características notables y únicas de composición florística y riqueza de especies (Miranda y Sharp, 1950; Rzedowski, 1996) y por su función protectora de la cabecera de cuencas hidrográficas (Vogelman, 1973).

Estudios realizados en BMM han documentado la variación de condiciones microambientales, estructura de la vegetación y composición florística en gradientes de interior del bosque-borde-exterior (Williams-Linera, 1993; Williams- Linera et al., 1998, Oosterhoorn y Kapelle, 2000); las condiciones del banco de semillas de especies arbóreas en fragmentos con diferentes niveles de perturbación (ÁlvarezAquino et al., 2005); el efecto de borde sobre la dispersión y remoción de semillas de encino en función del tipo y distancia del borde a lo largo del gradiente bosque-borde-pastizal (López-Barrera y Manson, 2006); el efecto de la distancia a partir del borde del bosque en la estructura sucesional de la vegetación de potreros abandonados de diferentes edades (Muñiz-Castro et al., 2006); los efectos del tipo de borde en el establecimiento de renuevo de encinos en el gradiente bosque-borde-exterior (López-Barrera et al., 2006); además de el daño por herbivoría en la regeneración de encinos en función del tipo de borde y estación del año (Reynoso y Williams-Linera, 2007).

La mayor parte de los estudios sobre fragmentación y efecto de borde en BMM, así como en otros bosques de niebla (Oosterhoorn y Kapelle, 2000) y selvas tropicales (Laurance y Bierregaard, 1997) se han realizado en áreas con procesos activos de deforestación. En el presente trabajo abordamos el caso de un área, la Estación Científica Las Joyas (ECLJ) en la Reserva de la Biosfera Sierra de Manantlán, Jalisco, donde 25 años de protección han favorecido la recuperación de la cobertura forestal (Jardel-Peláez, 2008). La variación de la composición de especies, estructura de la vegetación y condiciones microclimáticas y edáficas a través de bordes en un gradiente desde el interior de rodales de BMM a rodales de bosques de pino-encino (BPQ) y matorrales secundarios fue analizada con el propósito de contribuir al entendimiento de la influencia de las condiciones de borde en el proceso de regeneración del bosque mesófilo de montaña, como un elemento para evaluar los resultados de la protección del área y fundamentar prácticas de conservación y restauración.

En este estudio consideramos las diferencias en la vegetación y las condiciones ambientales en dos tipos de bordes: "bordes abruptos", i.e. BMM-claros con matorrales secundarios establecidos en claros desmontados para la agricultura y "bordes suaves" en la transición del BMM con BPQ que tienen antecedentes de explotación maderera e incendios forestales (Jardel-Peláez, 1991). Se han utilizado los términos borde pronunciado, duro o abrupto para describir límites con marcado contraste entre el hábitat original y el transformado y bordes suaves o difusos para el caso de transiciones graduales entre dos tipos de bosques o comunidades ecológicas diferentes (Wiens et al., 1985; Forman, 1995). Los límites naturales con frecuencia son bordes suaves o ecotonos a través de los cuales la transición en la composición y estructura de la vegetación entre clases de bosques es gradual (Lindenmayer y Fischer, 2006). Ambos tipos de borde pueden actuar como barreras o membranas permeables en su estructura y en la respuesta de las especies, por ejemplo generalistas versus especialistas de hábitat (Laurance et al., 2001; López-Barrera et al., 2005; 2006).

En el área de estudio el BMM está asociado con condiciones geoformológicas y edáficas donde se acumula humedad, como hondonadas, laderas bajas cóncavas y barrancas y han sido reemplazados en algunas porciones de su área de distribución potencial por matorrales o por BPQ como consecuencia de desmontes agrícolas, explotación maderera e incendios (Jardel-Peláez, 1991, Jardel-Peláez, et al. 2004a), dando lugar tanto a bordes suaves (BMM-BPQ) como abruptos (BMM-matorral-herbazal). Estudios previos realizados en el área (reseñados en Jardel-Peláez et al., 2004c) indican que las especies arbóreas del bosque mesófilo de montaña son capaces de colonizar el sotobosque de rodales adyacentes de BPQ y que existe una tendencia de reemplazo sucesional que puede llevar a la regeneración del BMM en sitios húmedos. Estas condiciones hacen de la ECLJ un sitio interesante para estudiar la variación de la vegetación a través de bordes del BMM en un paisaje forestal protegido donde la cobertura forestal está en proceso de recuperación.

Como hipótesis de trabajo se planteó que el cambio en la composición y diversidad de especies y las condiciones microclimáticas y edáficas debe ser más marcada en los bordes abruptos que en los suaves, y que en los primeros la colonización de especies del BMM en los rodales adyacentes estará más limitada por las condiciones ambientales que en los segundos.

\section{Materiales y métodos}

Área de estudio. La ECLJ se localiza en el suroeste del estado de Jalisco (19³4'14"'-19³7'30" Norte y 10414'49”$104^{\circ} 18^{\prime} 16^{\prime \prime}$ Oeste); su superficie es de 1,257 ha y su ampli- 


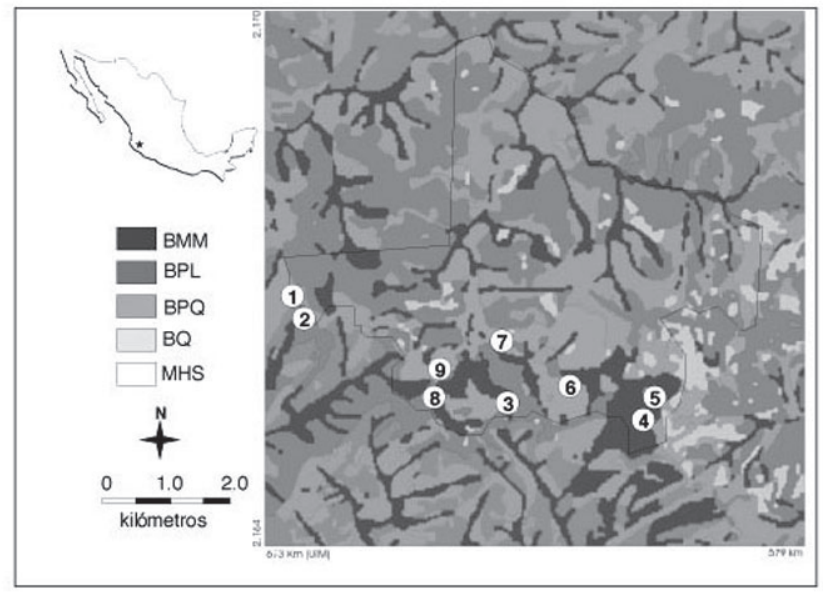

Figura 1. Localización del área de estudio y cobertura vegetal de la Estación Científica Las Joyas y sus alrededores. Tipos de vegetación: BMM, bosque mesófilo de montaña; BPL, bosque de pinolatifoliadas; BPQ, bosque de pino-encino; BQ, bosque de encino, y MHS, matorrales y herbazales secundarios. Los números indican la localización de los sitios de muestreo y corresponden a los del cuadro 1.

tud altitudinal de 1,540 a 2,242 m (Figura 1). El relieve es complejo y está formado por laderas y cimas de montaña, cañadas y hondonadas o depresiones (llamadas localmente "joyas", lo que dan su nombre a la localidad). El clima es templado subhúmedo, $\mathrm{Ca}\left(w_{2}\right)(w)(e) g$ basado en la clasificación de Köeppen modificada por García (1973); la temperatura media anual es de $15.5^{\circ} \mathrm{C}$ y la precipitación pluvial media anual es de 1,826 $\pm 94 \mathrm{~mm}$ (Jardel-Peláez et al., 2004b). El sustrato geológico corresponde a rocas ígneas extrusivas y la catena de suelos típica del área se caracteriza por suelos someros de perfil poco desarrollado, del orden de los inceptisoles (eutocrept dístrico), en las cimas; en las laderas los suelos corresponden al orden de los alfisoles (más profundos, con saturación de bases y perfil más diferenciado), que en el gradiente topográfico pasan de paleudalf típico en las laderas altas y medias, a paleudalf mólico en las laderas bajas; por último, en las cañadas y depresiones se encuentran ultisoles (palehumult típico), que son los suelos más desarrollados del área (Martínez-Rivera et al., 1993). La variación de la vegetación en el gradiente topográfico está asociada al cambio en la humedad y disponibilidad de nutrientes del suelo, con los pinares ocupando posiciones topográficas altas sobre inceptisoles y alfisoles y el BMM asociado a las cañadas y depresiones con alfisoles y ultisoles (Jardel-Peláez et al., 2004b).

La heterogeneidad de las condiciones fisiográficas, junto con el efecto de una larga historia de influencia humana a través de la agricultura, el pastoreo, la explotación maderera y los incendios forestales (Jardel-Peláez 1991), se reflejan en la variación de la cobertura vegetal, la diversidad de hábitat y la riqueza florística en un espacio relativamente reducido; se han registrado 850 especies de plantas vasculares en el área (Cuevas-Guzmán et al., 2004). La cubierta vegetal (Figura 1) está formada por BMM, bosques dominados por el género Pinus (BP) en los que se diferencian tres subtipos: bosque mixto de pino-latifoliadas, bosque de pino-encino y bosque de pino; bosque de encino, matorrales y herbazales secundarios, y vegetación de escarpes rocosos (Jardel-Peláez et al., 2004a).

Los desmontes agrícolas, la explotación maderera y los incendios forestales, han contribuido a reducir tanto al BMM como al BP, abriendo claros cubiertos actualmente por matorrales (Jardel-Peláez, 1991). En 1986, poco después de establecerse el área protegida, los bosques cubrían $76 \%$ de la ECLJ; en 2000 la cubierta forestal había aumentado a 95\% (Jardel-Peláez, 2008). Además del aumento de la cobertura forestal, y la formación de rodales de bosque mixto de pinolatifoliadas (una etapa en la sucesión BP-BMM), entre 1986 y 2000 se ha observado una tendencia hacia el aumento de la proporción interior-borde de los rodales y una reducción de la proporción de bordes abruptos (BMM-matorral) respecto a los bordes suaves (BMM-BP) (Santiago-Pérez, 2006; Jardel-Peláez, 2008).

Utilizando un mapa de vegetación reciente (Jardel-Peláez et al., 2004a) y recorridos de campo se seleccionaron rodales de BMM de la microcuenca del arroyo Las Joyas con base en la presencia de fragmentos de bosque maduro rodeados por diferentes tipos de vegetación (Figura 1). Se identificaron dos tipos de bordes y se establecieron en total nueve parcelas de muestreo de $160 \times 10 \mathrm{~m}\left(1,600 \mathrm{~m}^{2}\right)$, cinco en el borde BMM con BPQ (bordes suaves o B-B) y cuatro en bordes de $\mathrm{BMM}$ con matorrales o herbazales secundarios (bordes abruptos o B-M). El eje largo de cada parcela se estableció perpendicular al gradiente hacia el interior del BMM $(-80 \mathrm{~m})$, pasando por el borde $(0 \mathrm{~m})$ hasta el interior de la vegetación adyacente $(+80 \mathrm{~m})$. El borde en los B-B se definió en función del cambio en la cobertura del dosel; esto es, en las condiciones del área de estudio es posible observar un cambio definido entre el dosel del BMM dominado por árboles latifoliados (mayor a 90\% de cobertura de copas) y el dosel del BPQ donde los pinos representan más del $80 \%$ de la cobertura de copas. En el borde B-M los $0 \mathrm{~m}$ se ubicaron entre los límites de la cobertura arbórea del BMM y el área abierta de los claros adyacentes (Figura 2). Un método similar ha sido utilizado en otros estudios (WilliamsLinera, 1993; Oosterhoorn y Kappelle, 2000; López-Barrera et al., 2005). Las parcelas se subdividieron en 16 unidades de $10 \times 10 \mathrm{~m}$ para facilitar el muestreo de la vegetación. Para el análisis de los datos de estructura y diversidad de especies cada 4 unidades contiguas de $10 \times 10 \mathrm{~m}$ se combinaron en cuadrantes de $10 \times 40 \mathrm{~m}\left(400 \mathrm{~m}^{2}\right)$; debido al tamaño de los fragmentos de BMM del área de estudio, se consideró arbitrariamente de $-80 \mathrm{~m}$ a $-40 \mathrm{~m}$ como "interior" y de $-40 \mathrm{~m}$ a $0 \mathrm{~m}$ como borde del BMM, $0 \mathrm{~m}$ a $+40 \mathrm{~m}$ como borde de la vegetación adyacente (BPQ o matorral) y de $+40 \mathrm{~m} \mathrm{a}+80 \mathrm{~m}$ 


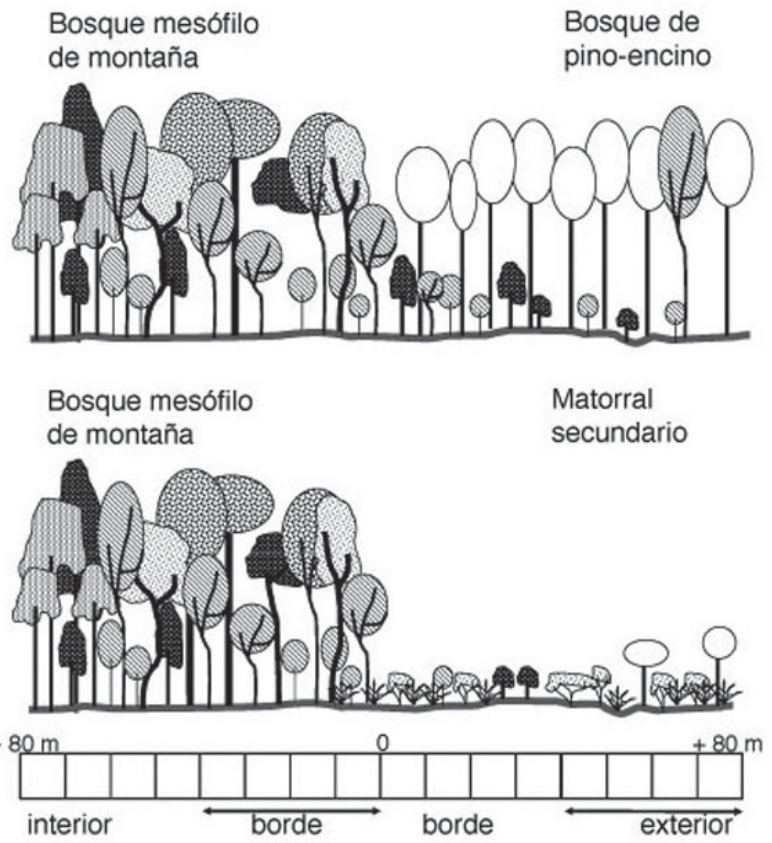

Figura 2. Perfiles de la vegetación a través de bordes suaves (BB) arriba, y bordes abruptos (B-M) abajo, mostrando en la parte inferior la forma de las parcelas de muestreo.

como "exterior" para los rodales de la vegetación adyacente (Figura 2).

En cada parcela se determinaron las condiciones físicas como altitud y pendiente promedio, exposición, geoforma (cima, ladera convexa o cóncava, o cañada) y la posición topográfica (alta, media o baja) del sitio respecto al cauce más próximo (Cuadro 1).

En todas las parcelas se midieron, registraron e identificaron todas las plantas leñosas con diámetro a la altura del pecho (DAP, medido a $1.3 \mathrm{~m}$ del suelo) $\geq 2.5 \mathrm{~cm}$ como estrato arbóreo y como estrato arbustivo-herbáceo a los latizales (con DAP $\leq 2.5 \mathrm{~cm}$ ) y plántulas (altura menor a 50 $\mathrm{cm})$. Se caracterizó la estructura de las parcelas mediante las variables abundancia, área basal por grupos de especies (pinos y latifoliadas) y se elaboraron gráficas de distribución de abundancia por clases diamétricas con cuatro categorías: $2.5-5 \mathrm{~cm},>5-<30 \mathrm{~cm},>30-60 \mathrm{~cm} \mathrm{y} \geq 60 \mathrm{~cm}$. Las especies del estrato arbustivo-herbáceo fueron contadas, identificadas y registradas dentro de 5 cuadros de $1 \mathrm{~m}^{2}$ distribuidos sistemáticamente cada $10 \mathrm{~m}$ a través de la parcela de 1,600 $\mathrm{m}^{2}$. Se recolectaron ejemplares botánicos, los cuales fueron identificados en forma crítica y quedaron depositados en el Herbario ZEA del Centro Universitario de la Costa Sur como material de referencia de la investigación. La nomenclatura de las especies se basó en Cuevas-Guzmán et al. (2004).

Para caracterizar la composición de especies y diversidad a través del borde en cada parcela se obtuvo el número de familias, géneros, especies y se calculó el índice de concentración de Simpson (Magurran, 2004); además se estimó el cambio en la composición de especies, utilizando como referencia el interior del BMM, con el índice de semejanza de Sørensen; todos los cálculos se realizaron con el programa EstimateS 7.5.1 (Colwell, 2005).

La variación en las condiciones de microclima a través del gradiente fue evaluada estimando la cobertura del dosel

Cuadro 1. Descripción de las condiciones de borde y topográficas de los sitios de estudio (el número de sitio corresponde con la localización en la figura 1).

\begin{tabular}{|c|c|c|c|c|c|c|}
\hline Sitio & $\begin{array}{l}\text { Vegetación } \\
\text { adyacente }^{(1)}\end{array}$ & $\begin{array}{l}\text { Tipo de } \\
\text { borde }^{(2)}\end{array}$ & $\begin{array}{l}\text { Altitud } \\
\text { media }(m)\end{array}$ & $\begin{array}{c}\text { Geoforma } \\
\text { y posición } \\
\text { topográfica }\end{array}$ & $\begin{array}{l}\text { Pendiente } \\
\text { media (\%) }\end{array}$ & Orientación e historia de uso \\
\hline 1. Cañada La Moza (CLM) & $\mathrm{BP}$ & $\mathrm{BB}$ & 1895 & LMC & 28 & N, exclusión pastoreo en 1986 \\
\hline 2. Puerto La Moza (PLM) & $\mathrm{BP}$ & BB & 1950 & LAC & 46 & N, exclusión pastoreo en 1986 \\
\hline 3. Tuna (TUN) & $\mathrm{BP}$ & BB & 1875 & LBC & 19 & $\begin{array}{l}\text { NO, sin fuego desde } 1979 \\
\text { exclusión pastoreo en } 1984\end{array}$ \\
\hline 4. San Campús (SNC) & $\mathrm{BP}$ & BB & 2100 & LAC & 55 & SO, incendio de copa en 1983 \\
\hline 5. Puerto San Campús (PSC) & $\mathrm{BP}$ & BB & 2050 & LMC & 26 & $\begin{array}{l}\text { O, incendio de copa en } 1983 \text {, } \\
\text { exclusión pastoreo en } 1984\end{array}$ \\
\hline 6. Zarzamoro (ZAR) & S-Z & $\mathrm{BC}$ & 1890 & Cñ & 23 & $\begin{array}{l}\mathrm{N} \text {, cultivo antiguo de Zea } \\
\text { diploperennis }\end{array}$ \\
\hline 7. Xilosuchitlán (XIL) & S-Z & $\mathrm{BC}$ & 1910 & LBC & 27 & $\begin{array}{l}\mathrm{N} \text {, cultivo antiguo de Zea } \\
\text { diploperennis }\end{array}$ \\
\hline 8. El Triguito 1 (ET1) & S-R & $\mathrm{BC}$ & 1895 & LMC & 20 & $\begin{array}{l}\text { N, exclusión pastoreo en 1984, } \\
\text { incendio superficial en } 1993\end{array}$ \\
\hline 9. El Triguito 2 (ET2) & S-R & $\mathrm{BC}$ & 1920 & LBCx & 17 & $\begin{array}{l}\text { N, exclusión pastoreo en } 1984 \text {, } \\
\text { incendio superficial en } 1993\end{array}$ \\
\hline
\end{tabular}

\footnotetext{
(1) BP, bosque de pino; S-Z, matorral secundario con Zea diploperennis; S-R, matorral secundario con zarzamoras (Rubus spp.)

${ }^{(2)} \mathrm{BB}$, borde suave bosque-bosque; $\mathrm{BC}$, borde abrupto bosque-claro

(3) $\mathrm{LMC}=$ ladera media cóncava; $\mathrm{LBC}=$ ladera baja cóncava; $\mathrm{LAC}=$ ladera alta cóncava; $C \tilde{n}=$ cañada; $\mathrm{LBC}=$ ladera baja convexa
} 
con densiómetro (Stumpf, 1993), cada $2.5 \mathrm{~m}$ de distancia en tres líneas, en los extremos y el centro de cada parcela. La temperatura y humedad relativa del aire se midieron con una estación climatológica portátil Krestel 4000 (1 m arriba del suelo) y la temperatura del suelo con un termómetro de suelos (de $15 \mathrm{~cm}$ de longitud); los registros se realizaron cada $20 \mathrm{~m}$ con respecto al borde del bosque $(0 \mathrm{~m})$ en cada parcela durante un día en los meses de enero, abril, julio y noviembre en tres horarios: mañana (0700-0800), al medio día (1200-1300) y por la tarde (1700-1800). Se estimó la oscilación entre los valores mínimos y máximos de cada variable, considerando que la variación en estos factores
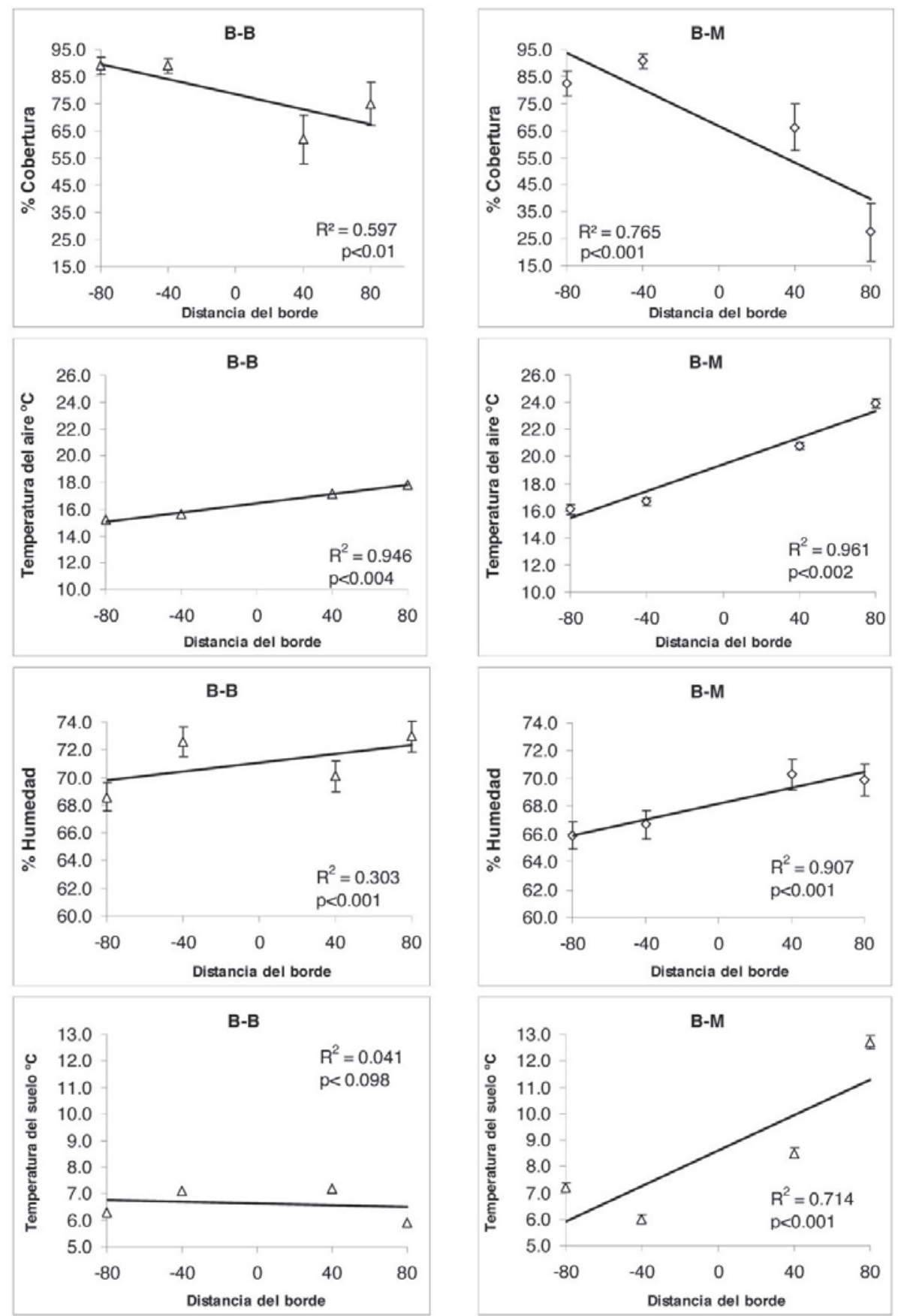

Figura 3. Cambios a través de los bordes B-B (izquierda) y B-M (derecha) de la cobertura del dosel (\%, arriba) y las variables del microclima: de arriba abajo oscilación en la temperatura del aire $\left({ }^{\circ} \mathrm{C}\right)$, humedad relativa $(\%)$ y temperatura del suelo $\left({ }^{\circ} \mathrm{C}\right)$. Se muestran valores medios y el error estándar de la media. 
ambientales es más crítica para las plantas que las condiciones promedio.

El muestreo del suelo se obtuvo en tres porciones de las parcelas: el interior de BMM (-70 a $-80 \mathrm{~m})$, en el borde $(0$ m) y exterior o vegetación adyacente $(+70 \mathrm{a}+80 \mathrm{~m})$. En cada porción dentro de cuadros de 10 x 10 m, se eligió un punto central y las cuatro esquinas para la obtención de cinco muestras hasta $30 \mathrm{~cm}$ de profundidad (sin hojarasca), las cuales se mezclaron formando una muestra combinada. Finalmente se determinaron los análisis físicos y químicos, humedad, $\mathrm{pH}$, capacidad de intercambio catiónico (CIC) y porcentaje de materia orgánica, por parte del Laboratorio de Suelos del Centro Universitario de Ciencias Biológicas y Agropecuarias (CUCBA), Universidad de Guadalajara.

Se realizaron análisis de todas las variables antes mencionadas, mediante la aplicación de pruebas comparativas de análisis de varianza para significancia estadística $\alpha=0.05$ para determinar la existencia o no de diferencias en atributos de riqueza, similitud florística y estructurales dentro y entre parcelas a través del borde y del gradiente bosqueborde-exterior; el cambio en las variables ambientales se evaluó mediante análisis de regresión (Zar, 1999). La variación de la composición de especies por estrato entre tipos de borde y el gradiente y su relación con las condiciones de microclima y suelos, se analizó mediante una ordenación polar (Bray y Curtis, 1957; Beals, 1984) con el programa PC-ORD 4.0 (McCune y Mefford, 1999), con datos de presencia y ausencia de las especies (transformados por el método de suavizamiento de Beals), distancia de Sørensen y varianza-regresión para la selección de los puntos extremos.

\section{Resultados}

Bordes suaves vs Bordes abruptos. Los valores de cobertura media del dosel fueron mayores en el borde del BMM tanto en los B-B $\left(89 \pm 3 \%, \mathrm{t}=7.51, P<0.001, \mathrm{~g} .1 .=38, \mathrm{R}^{2}=0.597\right)$, como en los B-M $(90.6 \pm 2.9 \%, \mathrm{t}=10.1, P<0.001$, g.l. $=31$, $\mathrm{R}^{2}=0.765$ ), el borde de bosque de pino fue de $61.9 \pm 9.1 \%$

Cuadro 2. Tendencias de las variables de respuesta en relación a la distancia del borde en bosque mesófilo de montaña en la Estación Científica Las Joyas, Sierra de Manantlán, México. Se muestran valores medios y error estándar de la media.

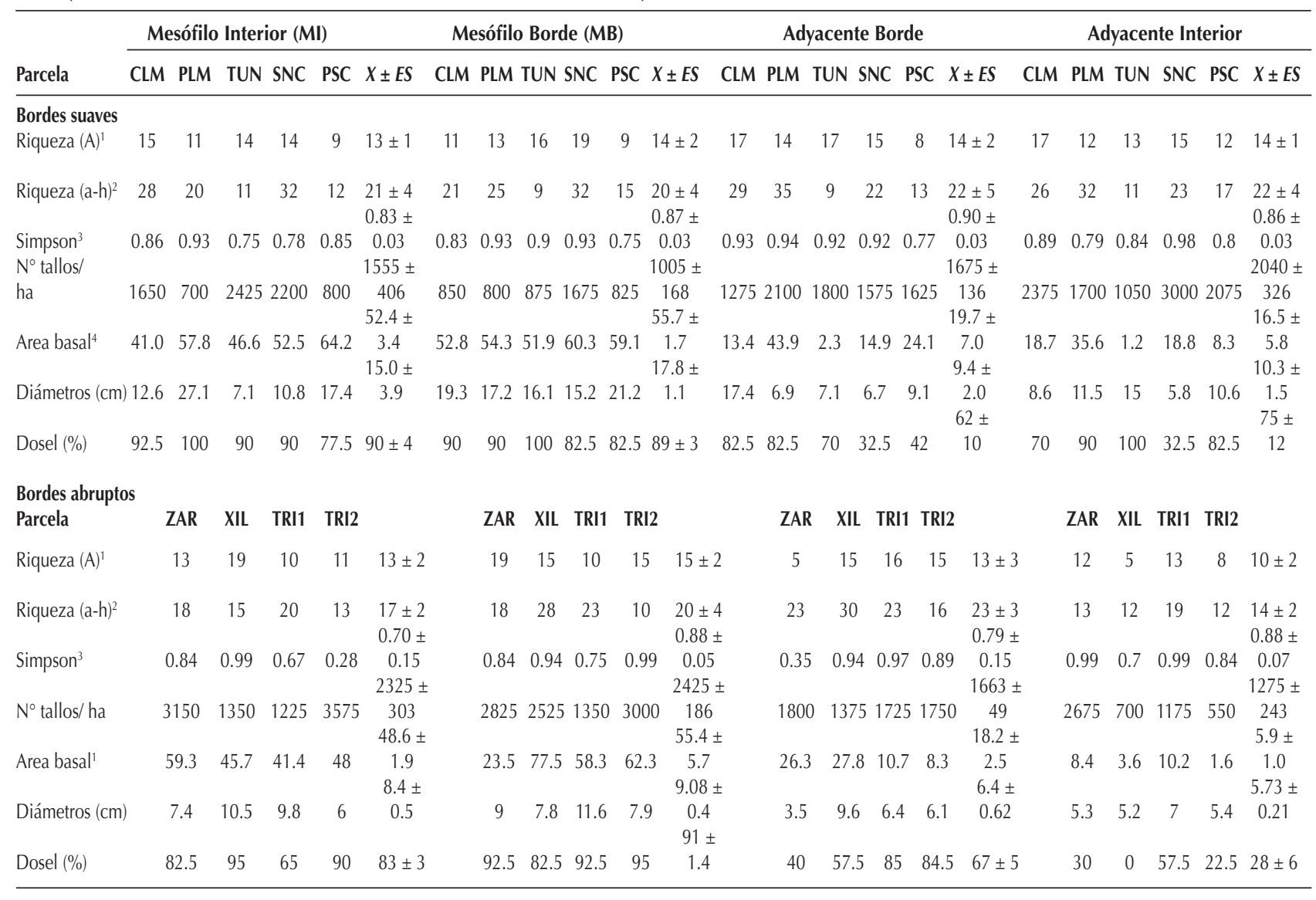

1. Riqueza especies arbóreas

2. Riqueza especies arbustivas y herbáceas

3. Indice de diversidad Simpson

4. $\mathrm{m}^{2} / \mathrm{ha}^{-1}$ 

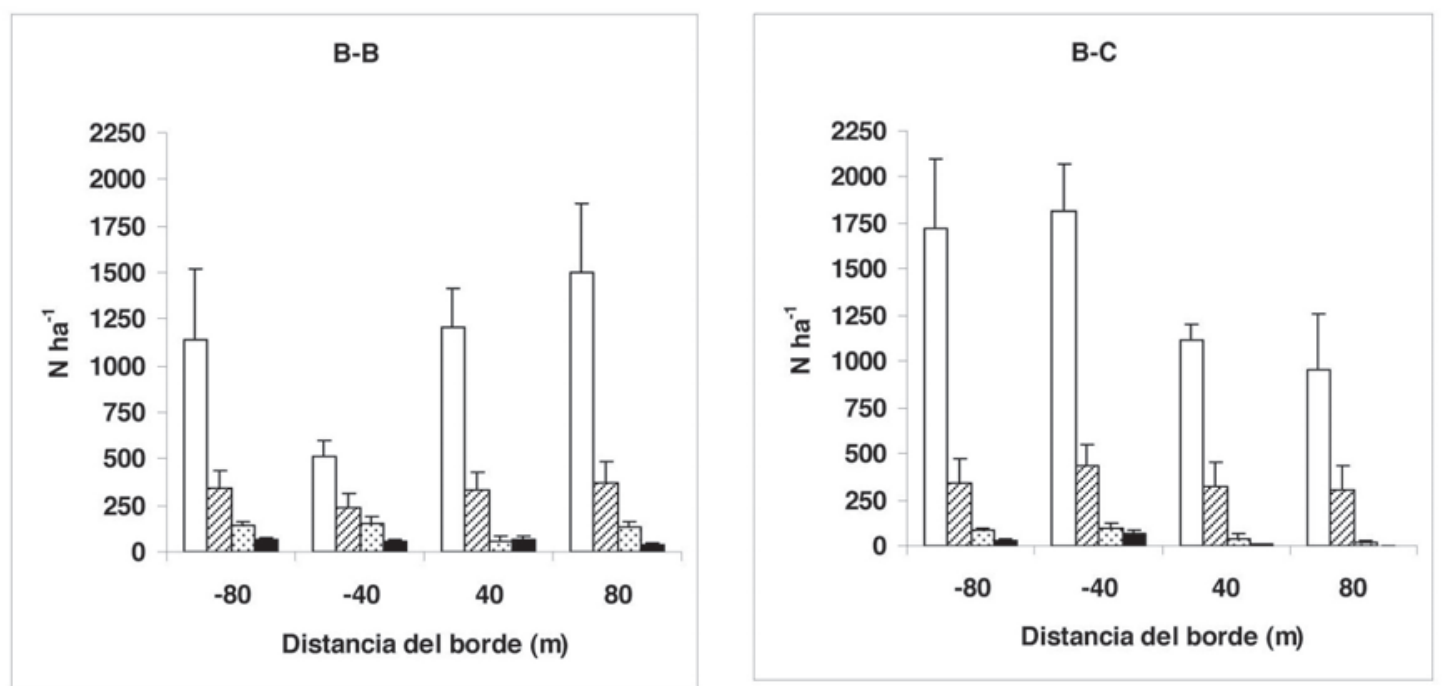

Figura 4. Cambio en la estructura de diámetros desde el interior del bosque mesófilo de montaña a la vegetación adyacente a través del borde (número promedio de individuos por ha \pm 1 error estándar). A la izquierda bordes B-B y a la derecha bordes B-M. Las categorías de diámetro son: $<5 \mathrm{~cm}$ (barras blancas), 6-30 cm (barras con diagonales), 31-60 cm (barras con puntos) y $>60 \mathrm{~cm}$ (barras negras).

y de $66.3 \pm 8.7 \%$ en el borde de los matorrales respectivamente (Figura 3).

Las diferencias en oscilación en la variables microclimáticas mostraron las siguientes tendencias (Figura 3): la temperatura del aire aumentó en los $\mathrm{B}-\mathrm{B}\left(\mathrm{de} 15.6^{\circ} \mathrm{C}\right.$ a $17.2^{\circ}$ C) $\left(\mathrm{t}=8.37, P<0.004, \mathrm{~g} .1 .=3, \mathrm{R}^{2}=0.94\right)$; en tanto los bordes abruptos registraron temperaturas más altas en los claros respecto a los bosques $\left(20.8^{\circ} \mathrm{C}\right.$ vs. $16.7^{\circ} \mathrm{C}, \mathrm{t}=9.91, P<0.002$, g.l. $=3, \mathrm{R}^{2}=0.96$ ). La humedad relativa osciló ligeramente a través de bordes suaves $(72.6$ a $70.1 \%, \mathrm{t}=12.47, P<0.001$, g.l. $\left.=5, R^{2}=0.303\right)$, mientras que en los $B-M$ se observó una mayor oscilación $(66.7$ a $70.3 \%, \mathrm{t}=52.72, P<0.001$, g.1. $=5$, $\left.\mathrm{R}^{2}=0.907\right)$ con respecto a los bosques. La oscilación de la temperatura del suelo no varió en los B-B $\left(7.1\right.$ a $7.2^{\circ} \mathrm{C}$, $\mathrm{t}=2.02, \mathrm{P}=0.098$, g.l $=5, \mathrm{R}^{2}=0.041$ ), pero aumentó significativamente en los $\mathrm{B}-\mathrm{M}\left(6.0\right.$ a $8.5^{\circ} \mathrm{C}, \mathrm{t}=15.38, P<0.001$, g.l. $=5, \mathrm{R}^{2}=0.714$ ).

La abundancia de árboles pequeños o juveniles (DAP $<5 \mathrm{~cm}$ ) del BMM fue menor en el borde B-B (510 árboles ha $\left.^{-1}\right)(F=20.24 ;$ g.l. $=3,12 ; P<0.001)$, mientras que en los bordes B-M (1819 árboles ha $\left.{ }^{-1}\right)$ fue mayor $(F=3.47$; g.l. $=$ 3, 16; $P=0.04)$, sin embargo en el borde B-B mostraron
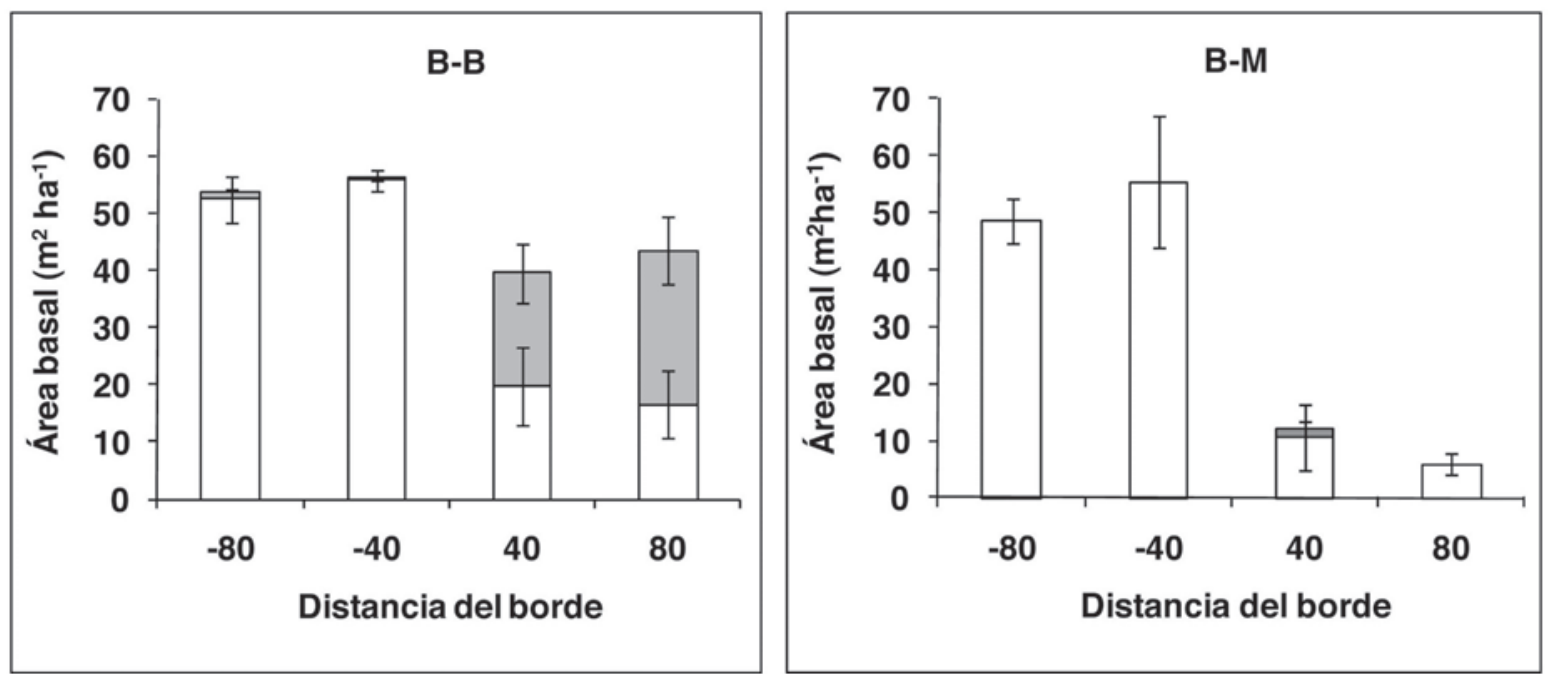

Figura 5. Área basal media de árboles de especies latifoliadas (barras blancas) y de árboles de especies de pinos (barras sombreadas) a través del borde en bordes suaves, B-B (izquierda) y bordes abruptos, B-M (derecha). 
incremento en densidad hasta $80 \mathrm{~m}$ al interior del BP(Figura 4, Cuadro 2). Los diámetros promedio de las parcelas MB en bordes suaves son mayores a los diámetros $\mathrm{MB}$ de bordes abruptos $(F=35.5 ;$ g.1.= 1,$8 ; P<0.001$; Cuadro2). La presencia de una alta densidad de árboles pequeños (2.5 - 5 cm DAP) en el BP adyacente a BMM (1210 a 1500 ár- boles ha-1 en B-B) se debió a la abundancia de latifoliadas establecidas en el sotobosque. Especies características del BMM como Carpinus tropicalis, Clethra fragrans, Cornus disciflora, Symplocos citrea y Zinowiewia concinna constituyen en promedio una alta proporción del área basal del borde $(47.8 \pm 12.0 \%)$ y el interior del BP $(38.4 \pm 11.5 \%)$
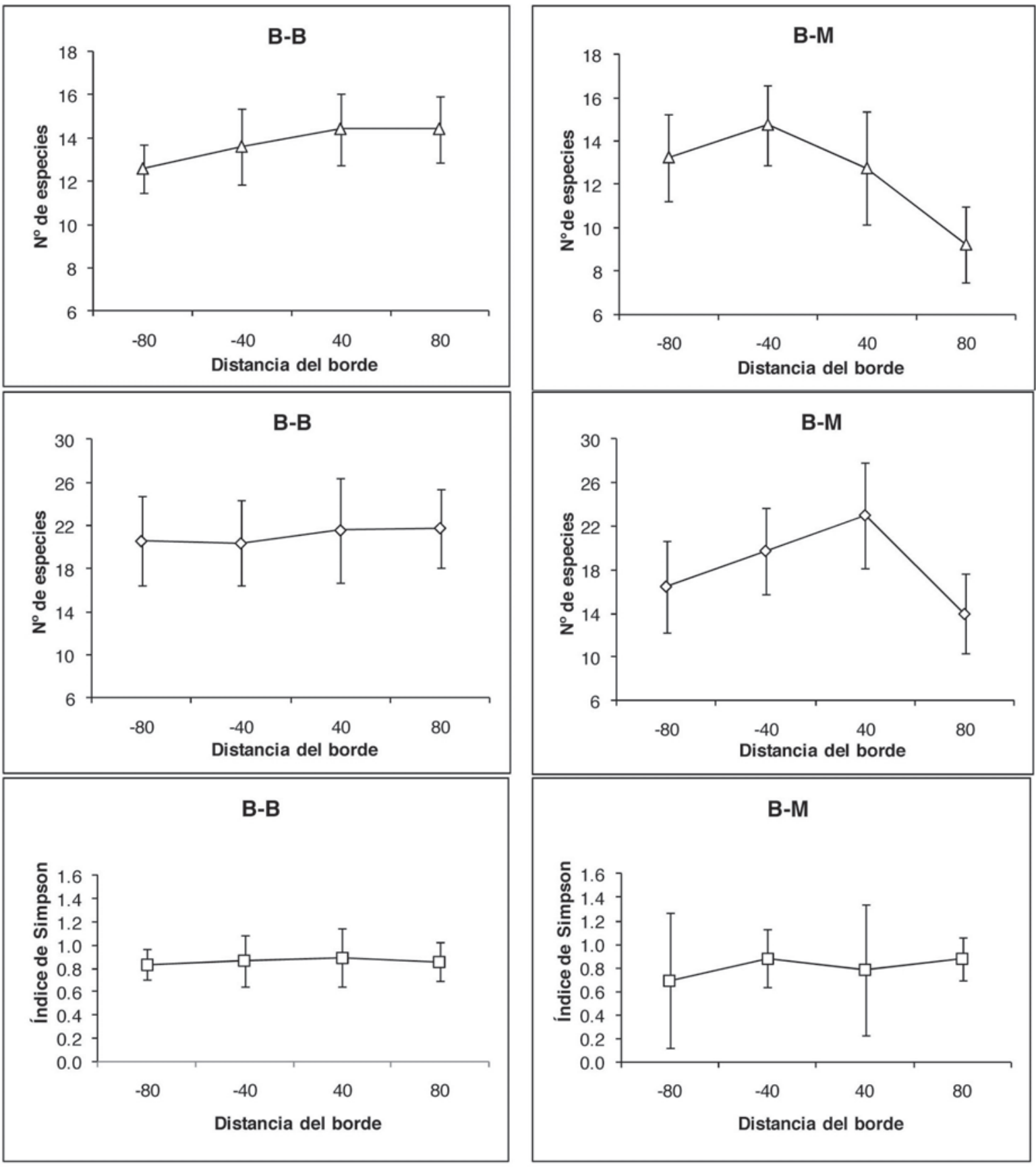

Figura 6. Cambio en la riqueza y diversidad de especies a través de bordes suaves, B-B (izquierda) y bordes abruptos, B-M (derecha); de arriba abajo: riqueza de especies arbóreas, riqueza de especies arbustivas y herbáceas, índice de diversidad de Simpson del estrato arbóreo. Se muestran valores medios y el error estándar de la media. 

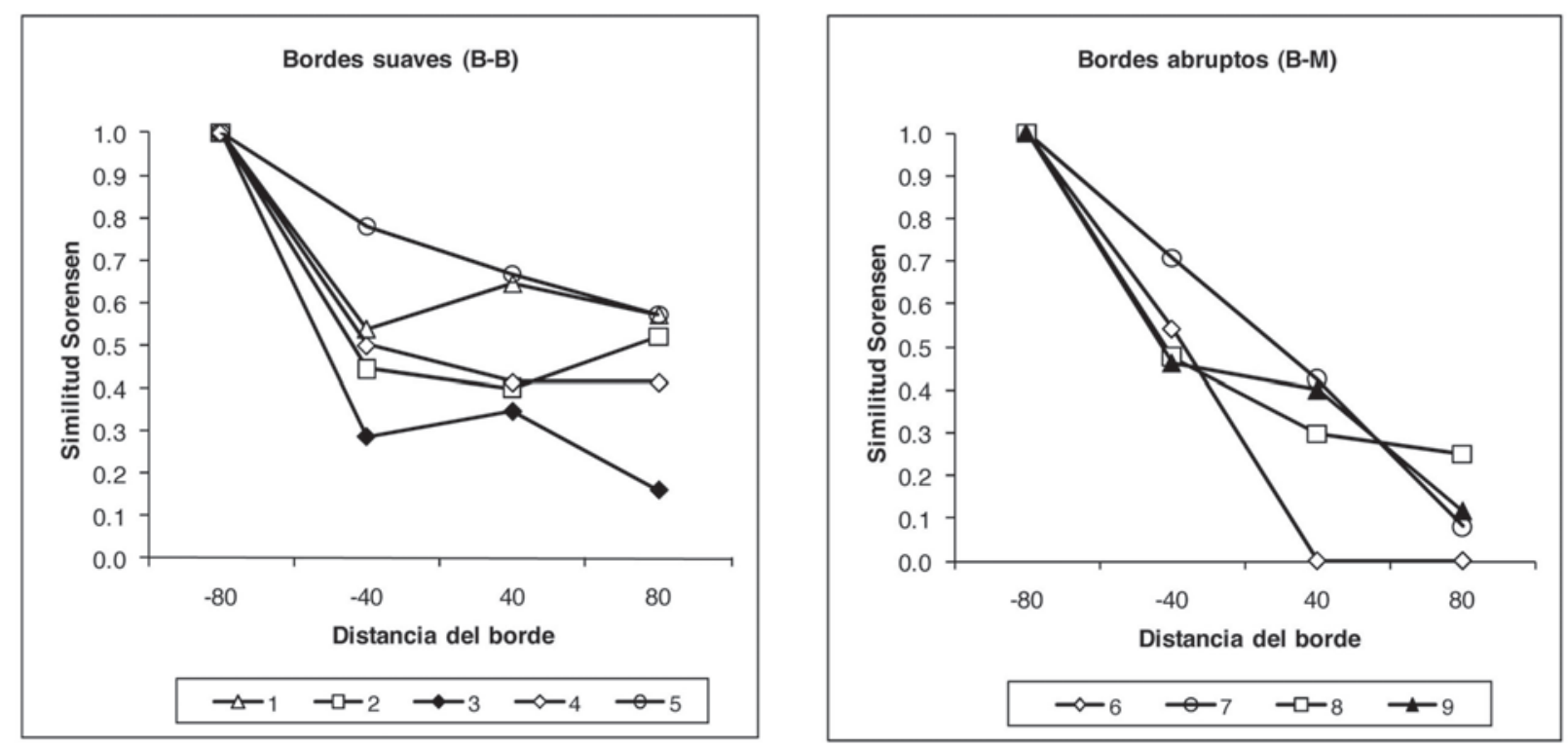

Figura 7. Cambio en el índice de similitud de Sorensen desde el interior del BMM (tomado como referencia) a la vegetación adyacente a través de bordes B-B (izquierda, número de sitio de muestreo del 1 al 5) y B-M (derecha, número de sitio de muestreo del 6 al 9).

(Figura 5, ver Apéndice). El área basal interior del BMM (MI) fue similar entre los sitios de ambos tipos de borde $(F$ = 0.79; g.l.= 1, 7; $P=0.40 ;$ Cuadro 2), en algunas parcelas pueden encontrarse pinos grandes aislados que contribuyen con 2\% del área basal (Figura 5).

En los matorrales y herbazales sólo se encontraron algunos árboles con DAP $>5 \mathrm{~cm}$ dispersos, en cambio los árboles pequeños (DAP $<2.5 \mathrm{~cm} ; 1113$ árboles ha-1 ) fueron abundantes en el borde con BMM y en el interior de los claros (956 árboles ha-1, Figura 4). Entre las especies incluidas como arbustos altos están Acaciella angustissima, Buddleja parviflora y Vernonia capreifolia, árboles indicadores de antecedentes de cultivo como tejocote (Crataegus pubescens) y durazno (Prunus persica), y ocasionalmente árboles jóvenes de Pinus douglasiana, Carpinus tropicalis, Cornus disciflora, Fraxinus uhdei y Persea hintonii característicos como especies colonizadoras en la regeneración de natural del BMM (Apéndice).

En total se registraron 172 especies de plantas vasculares (20\% de la flora vascular de la ECLJ), de 116 géneros y 62 familias (Apéndice). En el BMM se registró la mayor riqueza de especies (126), en comparación con el BP (84) y el matorral secundario (71). Los bordes B-B presentaron una mayor riqueza de especies (126) que los bordes B-M (104). Los bordes B-B presentaron mayor riqueza de especies exclusivas (63), los bordes B-M (44), mientras que en ambos tipos de borde se encontraron otras 63 especies. Algunas especies se encontraron exclusivamente en un tipo de vegetación: 52 en BMM, 17 en los matorrales y 10 en BP (Apéndice).

No se encontraron diferencias en la riqueza de especies entre bordes ni estratos (Figura 6), sólo en los bordes B-B la riqueza de especies arbóreas tendió a disminuir marcadamente en los claros $(F=4.04 ;$ g.1.= 1,$49 ; P=0.09)$. En ambos bordes se observó una ligera tendencia al aumento de la riqueza (B-B arbóreo, $P=0.811$; B-B estrato arbustivoherbáceo; $P=0.852)$. Los índices de diversidad mostraron valores similares en los B-B (Figura 6). Se observó también que los índices de diversidad tienden a ser más bajos y con mayor variación (i.e. errores estándar más altos) en el borde B-M que en el borde B-B (Cuadro 2).

Gradientes bosque - bordes - exterior. La variación en la semejanza de la composición de especies desde el interior del BMM (tomada como referencia) a través del borde hacia el interior de la vegetación adyacente, mostró un patrón diferente entre los bordes B-B y B-M (Figura 7): mientras que entre BMM y BP el reemplazo de especies a través del borde es gradual (de 0.51 en $\mathrm{MI}$ a 0.47 en PI), entre BMM y los claros se observó una pendiente pronunciada en los índices de semejanza (de 0.55 en MI a 0.28 en SB). En el gradiente a través del borde $\mathrm{B}-\mathrm{M}$ se encontraron diferencias significativas de los índices de semejanza $(F=9.38 ;$ g.1.=2, $4 ; P<0.006)$.

La distribución de diámetros del estrato arbóreo en todos los sitios presentó a lo largo del gradiente bosque-bordeexterior una forma de "J invertida", al disminuir el número de individuos conforme aumenta el diámetro de los árboles (Figura 4). En los B-B no hubo diferencia en el número total de árboles de las categorías de diámetro menores a 5 y mayores a $60 \mathrm{~cm}$ de DAP entre hábitat de BMM en los dos tipos de borde y entre BMM y BP (Cuadro 2). 

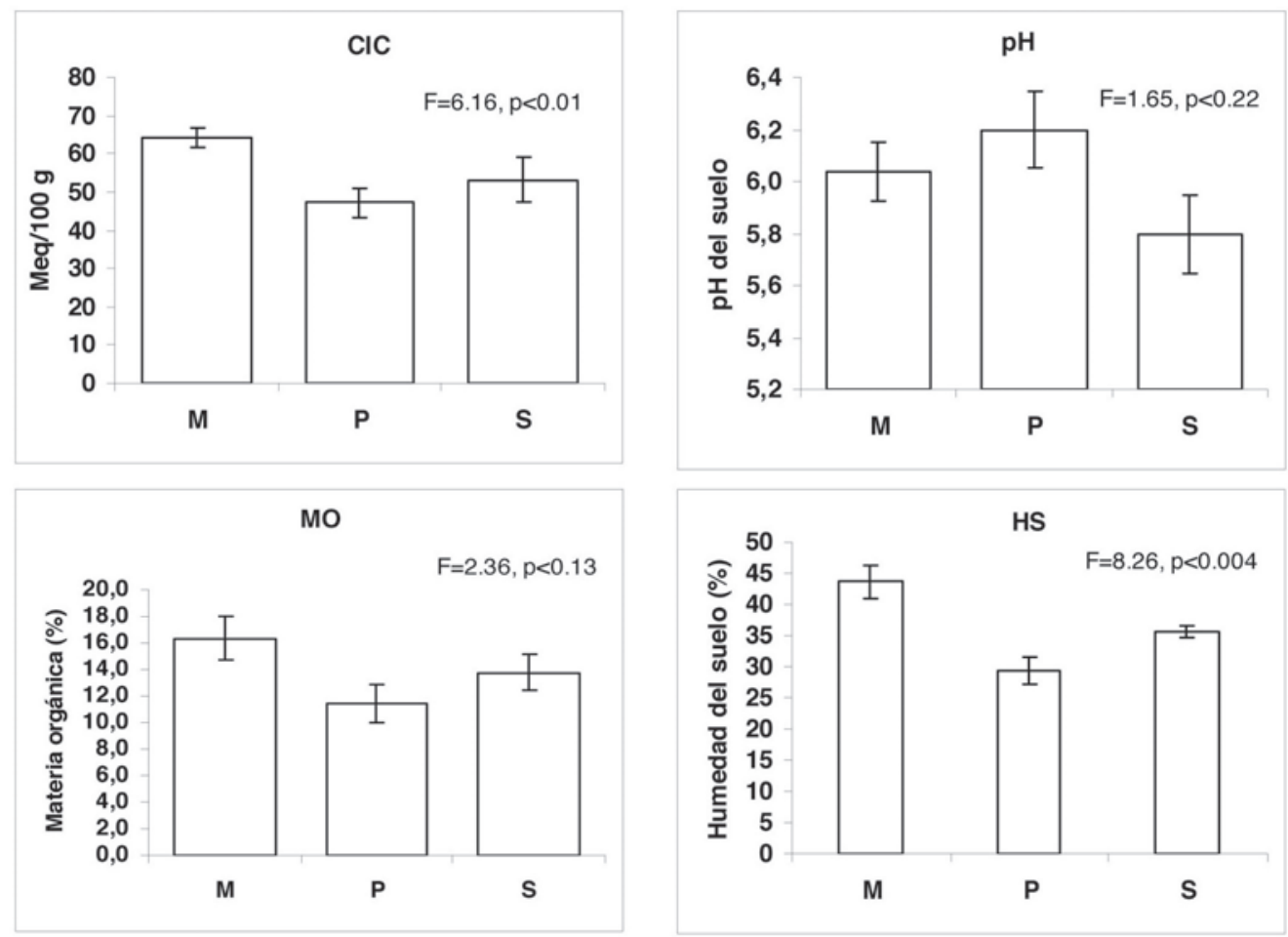

Figura 8. Variación observada en las condiciones del suelo en el interior del bosque mesófilo de montaña (M), bosque de pino (P) y matorrales-herbazales secundarios (S). Capacidad de intercambio catiónico (CIC), pH, materia orgánica (MO) y humedad del suelo (HS). Se muestran valores medios y el error estándar de la media.

Los suelos del BMM presentaron mayores valores de capacidad de intercambio catiónico $(\mathrm{CIC}=64.7 \mathrm{Meq} / 100 \mathrm{~g}$; $F=6.15$; g.l. $=2,15 ; P<0.01)$ y humedad respecto al BP y los matorrales secundarios $(\mathrm{HS}=43.7 \% ; F=8.26 ;$ g.l. $=2$, $15 ; P<0.003)$. No se observó variación entre los valores de medios del pH y la materia orgánica (Figura 8). El BMM tendió a ser ligeramente más ácido (6.04) y con mayor materia orgánica $(16.33 \%)$ respecto al BP, pero con menor acidez que los matorrales secundarios (Figura 8).

Los resultados de la ordenación de los sitios en función de la presencia-ausencia de especies mostraron un patrón similar para los estratos arbustivo-herbáceo y arbóreo (Figura 9): los bosques se separan de los matorrales a lo largo del eje 1 (71.7\% de la variación en la ordenación de los árboles y 77.14\% en la de arbustos y hierbas) y entre sí a lo largo del eje $2(18.45 \%$ de la varianza en la ordenación de los árboles y $15.1 \%$ en la de arbustos y hierbas). En la ordenación del estrato herbáceo-arbustivo (Figura 9b), se observó que tanto el interior como el borde de BP en el sitio PSC, afectado por un incendio severo en 1988, se separan marcadamente de los otros sitios de bosque.

La variación en la composición del estrato herbáceo-arbustivo en el eje 1 representa un gradiente de microclima, ya que se relaciona con la cobertura de $\operatorname{dosel}\left(r^{2}=0.52 ; F=\right.$
37.37; $P<0.001)$ y con la oscilación de la temperatura del aire $\left(r^{2}=0.22 ; F=9.38, P<0.004\right)$. Para el estrato arbóreo, el eje 1 se relacionó también con un gradiente de microclima, al mostrar relación con la cobertura del dosel $\left(r^{2}=0.37, F=\right.$ $21.85, P<0.001)$ y la oscilación térmica $\left(r^{2}=0.34, F=17.23\right.$, $P<0.001)$, mientras que el eje 2 se relacionó con un gradiente edáfico en el que la capacidad de intercambio catiónico fue la variable mejor correlacionada $\left(r^{2}=0.29\right.$, $F=13.61, P<0.001)$.

\section{Discusión}

La oscilación en las variables del microclima fueron mayores en los bordes abruptos que en los bordes suaves (Figura 3), por lo tanto los factores ambientales son más extremos y limitantes para el establecimiento de la regeneración de especies arbóreas del BMM (mayor oscilación en temperatura del aire, humedad relativa y humedad del suelo), además de la competencia por la alta densidad de arbustos y herbáceas en los matorrales, también parece estar limitada por la presencia de especies dominantes, como Zea diploperennis (sitios ZAR y XIL) y Rubus spp. (sitios TRI1 y TRI2), condiciones similares del microclima y de competencia por especies herbáceas y arbustivas, se han observado en otros 

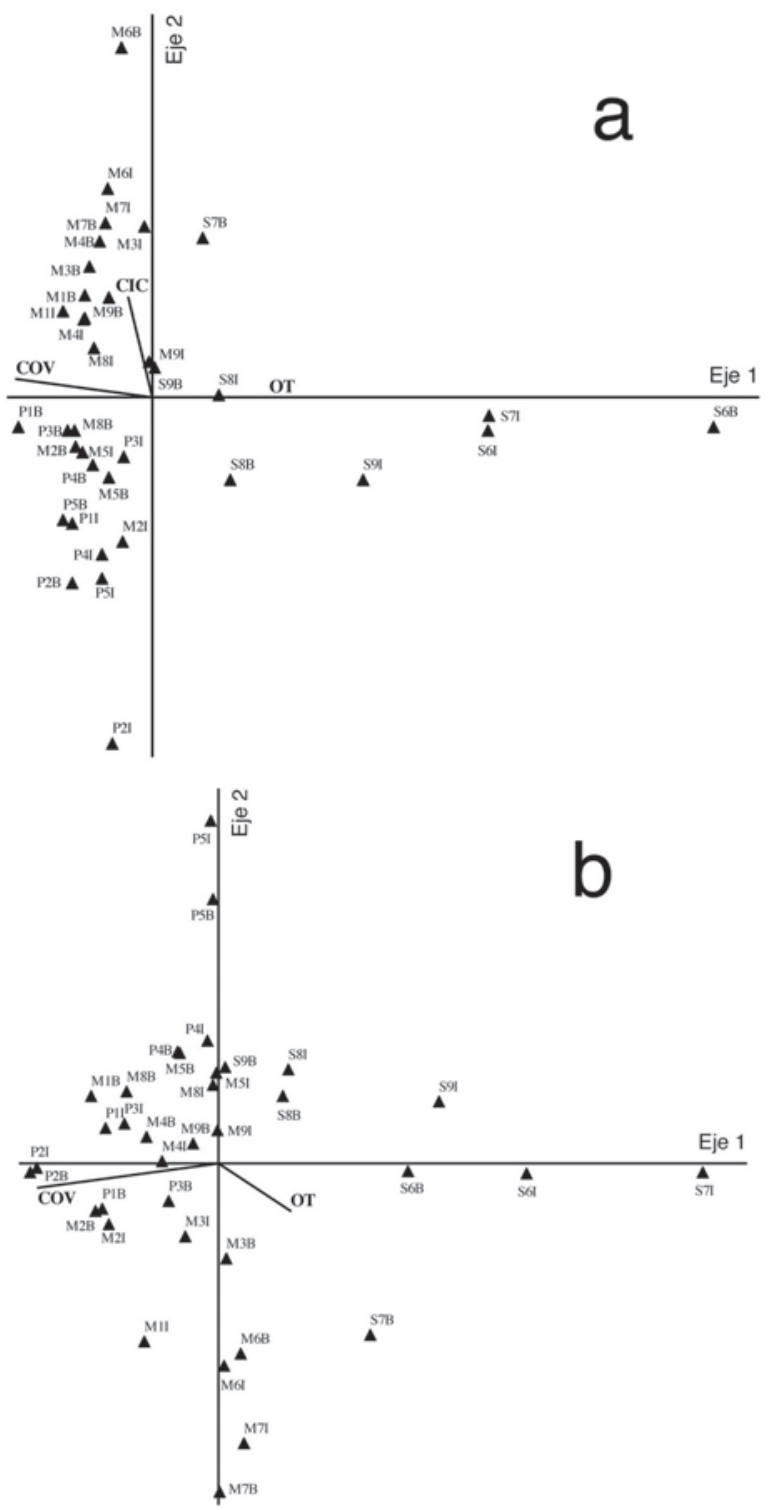

Figura 9. Ordenación de Bray y Curtis de los sitios en función de la presencia-ausencia de especies arbóreas (a) y herbáceas y arbustivas (b). Clave de los sitios: tipo de vegetación (M, bosque mesófilo de montaña; $\mathrm{P}$, bosque de pino; $\mathrm{S}$, matorrales secundarios), número de sitio y condición (I, interior; $\mathrm{B}$, borde). Se muestran las relaciones significativas con las variables ambientales $(\mathrm{COV}$, cobertura del dosel; OT, oscilación de la temperatura del aire; CIC, capacidad de intercambio catiónico del suelo).

trabajos (Williams-Linera et al., 1998; Gehlhausen et al., 2000; Oosterhoon y Kapelle, 2000). Valores más altos en la humedad relativa registrada en B-M, puede relacionarse con las condiciones topográficas en los sitios ZAR y XIL localizados en condiciones de cañada o ladera baja cóncava, respectivamente (Cuadro 1), además sus bordes están delimitados por el arroyo Las Joyas, el cual ejerce influen- cia en la humedad ambiental; mientras que los sitios TRI1 y TRI2 son claros grandes rodeados por bosque en laderas medias a bajas en exposición norte, que de alguna manera son claros protegidos por la cortina arbórea y la sombra orográfica. En este contexto, Gehlhausen et al.,( 2000) en su estudio demostraron que en los bosques deciduos de Norteamérica, la humedad relativa es la variable con mayor profundidad en la influencia de borde, hasta $80 \mathrm{~m}$ relacionada a la exposición oeste y la mayor amplitud de borde sobre exposiciones sur y oeste como respuesta al porcentaje de apertura del dosel.

El BMM en contacto con matorrales presentó mayor abundancia de árboles pequeños que en el borde con BPQ, lo cual puede explicarse por el efecto de la entrada de luz en la regeneración de plantas del sotobosque como se ha observado en otros estudios (Williams-Linera, 1993; WilliamsLinera et al., 1998; Gehlhausen et al., 2000; Oosterhoorn y Kappelle, 2000), esto difiere de lo encontrado en otros trabajos donde los bordes suaves registraron mayor densidad de plántulas (López-Barrera y Newton, 2005; GuzmánGuzmán y Williams-Linera, 2006). En tanto la cobertura relativamente alta en los bordes de los matorrales-herbazales secundarios indica la presencia de un frente de colonización de árboles desde el BMM adyacente.

De acuerdo con los resultados de la ordenación, la composición de especies tanto en el estrato arbóreo como arbustivo se correlacionó principalmente con la oscilación de temperatura del aire relacionada con la cobertura del dosel; sin embargo sólo el estrato arbóreo correlacionó también un gradiente edáfico (CIC). Las condiciones de gradientes ambientales a través de los bordes, al igual que lo muestran otros trabajos en bosques nubosos (Williams-Linera, 1993, Williams-Linera et al., 1998; Oosterhoorn y Kappelle, 2000), bosques tropicales húmedos (Laurance et al., 1997) y bosques subcaducifolios (Gehlhausen et al., 2000), indican que la distribución de las especies implican el análisis a mayor profundidad de interacciones y cambios de la composición a nivel comunidades. Sin embargo, recientes aportaciones para la regeneración de encinos realizadas por López-Barrera et al., (2007) indican que la dinámica de claros derivados de agricultura en baja escala y su subsecuente abandono en los Altos de Chiapas, pueden causar la variación de dispersión de semillas de encino por la combinación de factores que incluyen el contraste del borde, la ocurrencias de años semilleros, la cubierta vegetal de los claros y las fluctuaciones de la densidad de pequeños mamíferos. Así mismo, López-Barrera et al., (2007) enfatizan que el gradiente ambiental a través de los bordes con diferentes contrastes no es uniforme y que el reclutamiento de encinos parece depender principalmente de las respuestas específicas de las especies a la variación en disponibilidad de luz.

Las condiciones de borde en el área de estudio han sido favorables para algunas especies arbóreas con altas capaci- 
dades (dispersión y establecimiento) para utilizar la matriz circundante como las especies anemócoras: Carpinus tropicalis, Fraxinus uhdei, Tilia mexicana y las especies endozoocoras: Cornus disciflora, Styrax ramirezii y Zinowiewia concinna. Estas especies se destacan como colonizadoras en los bordes avanzando dentro del bosque de pino y los matorrales adyacentes en condiciones geomorfológicas y edáficas de sitios que en el pasado estuvieron ocupadas por el BMM (Jardel-Peláez et al., 2004a, este patrón de sucesión del bosque corresponde con los descritos en otros estudios (Saldaña-Acosta, 2001; Muñiz-Castro et al., 2006).

Los resultados obtenidos en cuanto al establecimiento de especies latifoliadas tanto en el sotobosque de los pinares como en los matorrales secundarios, concuerdan con la hipótesis del reemplazo sucesional del bosque de pino por el BMM sustentada en estudios previos en el área de estudio sobre la sucesión y la dinámica del paisaje (Jardel-Peláez et al. 2004a, 2004c), en la cual se esperaría que las especies del BMM estén colonizando el borde suave más marcadamente que el borde abrupto en ausencia de perturbaciones frecuentes, en suelos húmedos y relativamente fértiles (alfisoles), sobre geoformas predominantemente cóncavas.

La composición de especies en el área de estudio mostró una tendencia a ser una mezcla de especies que cambia en cuanto abundancia y estructura dependiendo del tipo de borde y con respecto al interior del bosque (Ver Apéndice, 55 especies en B-B versus 50 especies en B-M). Los índices de diversidad fueron similares en B-B y el reemplazo de especies fue gradual en la transición del BMM al BPQ, mientras que en la transición del BMM y los claros el cambio es contrastante y marcado (Figura 6). Si bien la media de la riqueza de especies varió poco entre sitios, tipos de vegetación y tipos de borde, las diferencias observadas en la composición se reflejaron en la baja semejanza entre tipos de borde. En los bordes suaves los valores de semejanza de Sørensen entre el interior y el borde de BMM variaron de 0.29 a 0.54 (Figura 7); solo el sitio PSC registró una similitud alta (0.78) entre interior - borde de BMM, debido a su afectación por un incendio de copa en 1988. Estas diferencias entre los dos tipos de borde se explican porque especies del BMM se encuentran en el sotobosque de los pinares y ambos tipos de vegetación comparten 31 especies, mientras que el borde B-M es más contrastante y solo se comparten 11 especies entre el BMM y los matorrales.

Una característica del BMM es la heterogeneidad de su composición florística; a pesar de su extensión reducida en el país $(\approx 1 \%$ de la superficie forestal del país) incluye el $10 \%$ de la flora vascular de México (Rzedowski, 1996), estudios anteriores en el BMM de la Sierra de Manantlán han indicado una alta diversidad $\beta$ (Santiago-Pérez y JardelPeláez, 1993).

A diferencia de las condiciones de áreas donde hay procesos activos de deforestación y fragmentación, donde la configuración de bordes abruptos mantenidos por la ac- tividad humana constituye el componente dominante del paisaje como se reporta en otros trabajos (Laurance et al., 1997; Williams-Linera et al., 1998; Oosterhoorn y Kappelle, 2000; Harper et al., 2005), en el área de estudio se presenta una tendencia a la recuperación de la cobertura forestal debido a las acciones de conservación puestas en práctica (Jardel-Peláez, 2008).

La conservación y restauración del BMM es una prioridad en la RBSM (INE, 2000). Como lo muestran los resultados obtenidos, se trata del tipo de vegetación con mayor riqueza de especies en el área de estudio (Ver Apéndice). Una manera de propiciar la restauración del BMM es eliminar los factores que interrumpen los procesos de regeneración de este tipo de vegetación, como los incendios y el apacentamiento de ganado, el cual ha sido el enfoque de manejo aplicado en la ECLJ (Jardel-Peláez, 2008). En tres sitios (TUN, ZAR y XIL), que han estado protegidos por más de 30 años, se encontró una alta densidad de plántulas y latizales de especies latifoliadas del BMM en el sotobosque del pinar; en contraste en los sitios PLM y PSC, afectados por incendios de copa en 1988 y 1983 respectivamente (Jardel-Peláez, 1991), el sotobosque es más abierto y está dominado por arbustos y herbáceas (Cuadro 2). Los incendios superficiales son un factor importante que arresta la sucesión de BPQ a BMM en el área de estudio, deteniendo el establecimiento de árboles latifoliados (Jardel-Peláez et al. 2004c).

En los bordes suaves se encontró que las especies arbóreas del BMM se están estableciendo en el sotobosque de los pinares, como predice la hipótesis sobre la sucesión pino-latifoliadas a partir de los estudios realizados en el área (Jardel-Peláez et al., 2004a). Es posible resaltar a escala del paisaje la relación funcional que existe entre distintos tipos de vegetación o hábitat (en este caso BMM y BPQ), como componentes de un mismo ecosistema que interactúan y que no son entidades que puedan ser separadas cuando se trata de establecer estrategias de gestión con fines de aprovechamiento, conservación o restauración (Santiago-Pérez, 2006; Jardel-Peláez, 2008). En este sentido Gehlhausen et al., (2000) indicaron que el tamaño y el potencial de amortiguamiento del hábitat adyacente debe ser considerado cuando se planifica la protección de bosques.

El manejo de un área protegida como la ECLJ plantea otros problemas para definir estrategias adecuadas de gestión. En el caso de los matorrales, la regeneración del BMM crea un conflicto con la conservación de un hábitat en el que se encuentra un componente importante de la biodiversidad del área, incluyendo plantas endémicas como Zea diploperennis, otras 81 especies vegetales exclusivas de áreas abiertas (Cuevas-Guzmán et al., 2004) y numerosas especies de aves residentes y migratorias (Santana-Castellón, 2000). El éxito en la recuperación de cobertura forestal en el área de estudio debido a su protección, implica ahora considerar el mantenimiento a largo plazo de algunos claros como parte de la estrategia de conservación de la biodiver- 
sidad en la ECLJ y sus alrededores, basada en mantener el mosaico dinámico de tipos de hábitat a escala del paisaje (Jardel-Peláez, 2008).

\section{Agradecimientos}

Esta es una contribución al programa de investigación del Grupo Manantlán de la Red Mexicana de Investigación Ecológica a Largo Plazo (MEXLTER) y es producto de la colaboración entre los Cuerpos Académicos de Conservación y Fomento Forestal (UDG-CA-537) y Manejo Forestal (UDGCA-315). Fue financiado por la Universidad de Guadalajara a través del CUCBA y del CUCSUR. Fue muy valioso el apoyo del personal de la ECLJ, así como de los voluntarios que participaron en el trabajo de campo. El manuscrito ha sido mejorado en gran medida por los comentarios de dos revisores anónimos y la editora del BSBM. La primera autora dedica a la memoria de su madre Tania Pérez Lemus $(\dagger)$ todos sus anhelos.

\section{Literatura Citada}

Alvarez-Aquino C., Williams-Linera G. y Newton A.C. 2005. Disturbance effects on the seed bank of Mexican cloud forest fragments. Biotropica 37:337-342.

Beals E.W. 1984. Bray-Curtis ordination: an effective strategy for analysis of multivariate ecological data. Advances in Ecological Research 14:1-55.

Bray J.R. y Curtis J.T. 1957. An ordination of upland forest communities of southern Wisconsin. Ecological Monographs 27:325-349.

Broadbent E.N., Asner G.P., Keller M., Knapp D.E., Oliveira P.J.C. y Silva J.N. 2008. Forest fragmentation and edge effects from deforestation and selective logging in the Brazilian Amazon. Biological Conservation 141:1745-1757.

Challenger A. 1998. Utilización y conservación de los ecosistemas terrestres de México. Pasado, presente y futuro. Comisión Nacional para el Conocimiento y Uso de la Biodiversidad, Instituto de Biología (UNAM) y Agrupación Sierra Madre A. C. México, D. F.

Colwell R.K. 2005. EstimateS Version 7.5.1. Statistical estimation of richness and shared species from samples. Disponible en linea: <http://viceroy.eeb.uconn.edu/estimates>

Cuevas-Guzmán R., Koch S., García-Moya E., Nuñez-López N.M. y Jardel-Peláez E.J. 2004. Flora vascular de la Estación Científica Las Joyas. En: Cuevas-Guzmán R. y Jardel-Peláez E.J. Eds. Flora y Vegetación de la Estación Científica Las Joyas, pp.117176, Universidad de Guadalajara, Guadalajara, Jalisco.

Fahrig L. 2003. Effects of habitat fragmentation on biodiversity. Annual Review of Ecology, Evolution and Systematics 34: 487515.

Forman R.T. 1995. Land mosaics: the ecology of landscapes and regions. Cambridge University Press, Nueva York.

García E. 1973. Modificaciones al sistema de clasificación climática de Köeppen Instituto de Geografía, Universidad Nacional Autónoma de México, México D.F.

Gehlhausen S.M., Schwartz M.W. y Augspurger C.K. 2000. Vegetation and microclimatic edge effects in two mixed-mesophytic forest fragments. Vegetatio 147:21-35.

Guzmán-Guzmán J. y Williams-Linera G. 2006. Edge effect on acorn removal and oak seedling survival in Mexican lower montane forest fragments. New Forests 31:487-495.

Harper K.A., Macdonald S.E., Burton P.J., Chen J., Brosofske K.D., Saunders S.C., Euskirchen E.S., Roberts D., Jaiteh M.S. y Essen P. 2005. Edge influence on forest structure and composition in fragmented landscapes. Conservation Biology 19:768782.

INE [Instituto Nacional de Ecología]. 2000. Programa de Manejo de la Reserva de la Biosfera Sierra de Manantlán. Secretaría de Medio Ambiente, Recursos Naturales y Pesca. Instituto Nacional de Ecología, México, D.F.

Jardel-Peláez E.J. 1991. Perturbaciones naturales y antrópicas de bosques subtropicales de montaña en Las Joyas, Sierra de Manantlán. Tiempos de Ciencia 22:9-26.

Jardel-Peláez E.J. 2008. Sucesión ecológica y restauración de bosques subtropicales de montaña en la Estación Científica Las Joyas, México. En: González-Espinosa M., Rey-Benayas J.M. y Ramírez-Marcial N. Eds. Restauración de Bosques en América Latina, pp. 77-97, Mundi-Prensa/ Fundación Internacional para la Restauración de Ecosistemas, México, D.F.

Jardel-Peláez E.J., Ezcurra E., Cuevas-Guzmán R., Santiago-Pérez A.L. y Cruz-Cerda P. 2004a Vegetación y patrones de paisaje. En: Cuevas-Guzmán R. y Jardel-Peláez E.J. Eds. Flora y Vegetación de la Estación Científica Las Joyas, pp.65-117, Universidad de Guadalajara, Guadalajara.

Jardel-Peláez E.J., Martínez-Rivera L.M., Ramírez R. J.M. y Partida-Lara D. 2004b Condiciones físico-geográficas de Las Joyas y sus alrededores. En: Cuevas-Guzmán R. y Jardel-Peláez E.J. Eds. Flora y Vegetación de la Estación Científica Las Joyas, pp.39-63, Universidad de Guadalajara, Guadalajara.

Jardel-Peláez E.J., Santiago-Pérez A.L., Cortés-Montaño C. y Castillo-Navarro F. 2004c. Sucesión y dinámica de rodales. En: Cuevas-Guzmán R. y Jardel-Peláez E.J. (Eds.) Flora y Vegetación de la Estación Científica Las Joyas, pp.179-203, Universidad de Guadalajara, Guadalajara.

Jardel-Peláez E.J., Santiago-Pérez A.L. y Muñoz-Mendoza M.E. 1993. El Bosque mesófilo de montaña de la Sierra de Manantlán. Tiempos de Ciencia 30:20-28.

Laurance W.F. y Bierregaard R.O. Eds. 1997. Tropical Forests Remnants: Ecology, Management and Conservation of Fragmented Communities. University of Chicago Press, Chicago.

Laurance W.F., Bierregaard R., Gascon C., Didham R., Smith A.P., Lynman A., Viana V., Lovejoy T.E., Sieving K., Sites J., Andersen M., Tocher M., Kramer E., Restrepo C. y Moritz C. 1997. Tropical forest fragmentation: Synthesis of a diverse and dynamic discipline. En: Laurance, W.F., y R. O. Bierregaard Eds. Tropical Forests Remnants: Ecology, Management and Conservation of Fragmented Communities, pp.502-514, University of Chicago Press, Chicago.

Laurance W.F., Didham R.K. y Power M.E. 2001. Ecological boundaries: a search for synthesis. Trends in Ecology and Evolution 16:70-71

Leopold, A. 1933. Game management. Charles Scribner's Sons. Nueva York.

Lindenmayer D.B. y Franklin J.F. 2002. Conserving Forest Biodiversity: a comprehensive multiscaled approach. Island Press, Washington, D.C.

Lindenmayer D.B. y Fischer J. 2006. Habitat Fragmentation and 
Landscape Change: an Ecological and Conservation Synthesis. Island Press, Washington, D.C.

López-Barrera F. y Newton A. 2005. Edge type effect on acorn germination of oak species in the Highlands of Chiapas, Mexico. Forest Ecology and Management 217: 67-79.

López-Barrera F., Newton A. y Manson R. 2005. Edge effects in a tropical montane forest mosaic: experimental tests of postdispersal acorn removal. Ecological Research 20:31-40.

López-Barrera F. and R.H. Manson. 2006. Ecology of acorn dispersal by small mammals in montane Forests of Chiapas, Mexico. En: Kappelle, M. Ed. Ecology and Conservation of Neotropical Montane Oak Forests, pp.165-174, Ecological Studies Vol. 185, Springer, Heidelberg.

López-Barrera F., Manson R. H., González-Espinosa M. y Newton A. 2006. Effects of the type of montane forest edge on oak seedling establishment along forest-edge-exterior gradients. Forest Ecology and Management 225:234-244

López-Barrera F., Manson R., González-Espinosa M. y Newton A.C. 2007. Effects of varying forest edge permeability on seed dispersal in a neotropical montane forest. Landscape Ecology 22:189-203.

Magurran A.E. 2004. Measuring biological diversity. Blackwell Publishing, Oxford.

Martínez-Rivera L.M., Delgado I.R. y Flores R.I. 1993. Suelos de la Estación Científica Las Joyas de la Reserva de la Biosfera Sierra de Manantlán. Agrociencia, 4:103-115.

Miranda, F. y Sharp A.J. 1950. Characteristics of the vegetation in certain temperate regions of Eastern México. Ecology 31: 313-333.

McCune B. y Mefford M.J. 1999. PC-ORD. Multivariate analysis of ecological data. Version 4. MjM Software Design, Gleneden Beach, Oregon.

Muñiz-Castro M.A., Williams-Linera G. y Rey-Benayas J.M. 2006. Distance effect from cloud forest fragments on plant community structure in abandoned pastures in Veracruz, Mexico. Journal of Tropical Ecology, 22:431-440.

Murcia C. 1995. Edge effect in fragmented forests: implications for conservation. Trends in Ecology and Evolution 10:58-62.

Oosterhoorn M. y Kappelle M. 2000. Vegetation structure and composition along an interior-edge-exterior gradient in a Costa Rican montane cloud forest. Forest Ecology and Management 126:291-307.

Reynoso J.A. y Williams-Linera G. 2007. Herbivory damage on oak seedlings at the edge of cloud forest fragments. Boletin de la Sociedad Botánica de México, 80:29-34.

Rzedowski J. 1996. Análisis preliminar de la flora vascular de los bosques mesófilos de montaña de México. Acta Botánica Mexicana 35:25-44.

Saldaña-Acosta M.A. 2001. Dinámica y patrones de establecimiento de especies de bosque mesófilo de montaña en la Sierra de Manantlán, Jalisco. Tesis de Maestría en Ciencias, Facultad de Ciencias, Universidad Nacional Autónoma de México, México, D.F. 87 pp.

Santana-Castellón E. 2000. Dynamics of understory birds along a cloud forest successional gradient. Tesis doctoral, University of Wisconsin-Madison, Wisconsin. 203 pp.

Santiago-Pérez A.L. 2006. Efecto de la fragmentación en la diversidad del bosque mesófilo de montaña, Sierra de Manantlán, México. Tesis de Maestría en Ciencias, Centro Universitario de Ciencias Biológicas y Agropecuarias, Universidad de Guadalajara, Guadalajara 78 pp.

Santiago-Pérez A.L. y Jardel-Peláez E.J. 1993. Composición de especies y estructura del bosque mesófilo de montaña de la Sierra de Manantlán, Jalisco-Colima. Biotam 5:13-26.

Shvidenko A., Barber C.V., Persson R., Gonzalez P., Hassan R., Lakyda P., McCallum I., Nilsson S., Pulhin J., van Rosenburg B. y Scholes B. 2005.Forest and woodland systems. En: Hassan R., Scholes R. y Ash N. Ecosystems and human well-being: current state and trends: faindings of the conditionand trendsworking group, pp.585-621, Millennium Ecosystem Assessment, Island Press, Washington, D.C.

Stumpf K.A. 1993. The estimation of forest vegetation cover descriptions using a vertical densitometer. Geographic Resource Solutions <www.grsgis.com/publications/saf_93.html> Consultado en 2009.

Vogelmann H.W. 1973. Fog precipitation in the cloud forests of eastern Mexico. Bioscience 23:96-100.

Wiens J. A., Crawford C. S. y Gosz J. R. 1985. Boundary dynamics: a conceptual framework for studying landscape ecosystems. Oikos 45:421-427.

Williams-Linera G. 1993. Vegetación de bordes de un bosque nublado en el Parque Ecológico Clavijero, Xalapa, Veracruz, México. Revista de Biología Tropical 41:443-453.

Williams-Linera G., Dominguez-Gastelú V. y García-Zurita M.E. 1998. Microenvironment and floristics of different edges in a fragmented tropical rainforest. Conservation Biology 12:10911102.

Williams-Linera G., Manson R.H. e Isunza-Vera E. 2002. La fragmentación del bosque mesófilo de montaña y patrones de uso del suelo en la región oeste de Xalapa, Veracruz, México. Madera y Bosques Vol. 8:73-89.

Zar J.H. 1999. Biostatiscal analysis. Prentice-Hall, Nueva Jersey.

Recibido: 8 de diciembre de 2008

Aceptado: 8 de agosto de 2009 
Apéndice. Listado de especies registradas por tipo de vegetación (BMM, bosque mesófilo de montaña; BP, bosque de pino; C, claros con matorrales secundarios), tipo de borde (BB, borde BMM-BP; BC, borde BMM-C), y forma de vida (FV): A, árbol; a, arbusto; h, hierba (ha, hierba anual; hp, hierba perenne; hp-e, hierba perenne epífita); L, liana.

\begin{tabular}{|c|c|c|c|c|c|c|}
\hline \multirow[t]{2}{*}{ FV } & \multirow[t]{2}{*}{ Familias y especies } & \multicolumn{3}{|c|}{$\begin{array}{c}\text { Tipo de } \\
\text { vegetación }\end{array}$} & \multicolumn{2}{|c|}{$\begin{array}{l}\text { Tipo de } \\
\text { borde }\end{array}$} \\
\hline & & BMM & BP & $\mathrm{C}$ & BB & BC \\
\hline A & $\begin{array}{l}\text { Actinidiaceae } \\
\text { Saurauia serrata DC. } \\
\text { Adiantaceae }\end{array}$ & 1 & & & 1 & \\
\hline hp & $\begin{array}{l}\text { Adiantum sp. } \\
\text { Anacardiaceae }\end{array}$ & & 1 & 1 & 1 & 1 \\
\hline $\mathrm{a}$ & $\begin{array}{l}\text { Rhus schmidelioides Schltdl. } \\
\text { Apiacecae }\end{array}$ & & 1 & & 1 & \\
\hline a & Coulterophytum sp nov. & 1 & & & & 1 \\
\hline hp & $\begin{array}{l}\text { Rhodosciadium pringlei S. Watson } \\
\text { Aquifoliaceae }\end{array}$ & 1 & & & 1 & \\
\hline A & $\begin{array}{l}\text { Ilex brandegeana Loes. } \\
\text { Araceae }\end{array}$ & 1 & 1 & & 1 & 1 \\
\hline $\mathrm{h}$ & $\begin{array}{l}\text { Arisaema macrospathum Benth. } \\
\text { Araliaceae }\end{array}$ & 1 & 1 & & 1 & \\
\hline A & Dendropanax arboreus (L.) Decne. y Planch. & 1 & 1 & 1 & 1 & 1 \\
\hline A & $\begin{array}{l}\text { Oreopanax echinops (Schltdl. y Cham.) Decne. y Planch. } \\
\text { Aspleniaceae }\end{array}$ & 1 & & & 1 & 1 \\
\hline hp & Asplenium auriculatum Sw. & 1 & & & & 1 \\
\hline hp & Asplenium cuspidatum Lam. & 1 & 1 & & 1 & \\
\hline hp & Asplenium monanthes L. & 1 & & & 1 & 1 \\
\hline hp & Asplenium sessilifolium Desv. & 1 & & & 1 & \\
\hline hp & Asplenium sp. 1 & 1 & & & & 1 \\
\hline hp & Asplenium sp. 2 & 1 & & & 1 & \\
\hline hp & Ctenitis equestris ((Kunze) Ching. & 1 & & 1 & & 1 \\
\hline hp & Diplazium lonchopyllum Kunze & 1 & & & & 1 \\
\hline hp & $\begin{array}{l}\text { Phanerophlebia nobilis (Schltdl. y Cham.) Presl. } \\
\text { Asteraceae }\end{array}$ & 1 & & & 1 & \\
\hline $\mathrm{L}$ & Archibaccharis schiedeana (Benth.) J.D. Jacks & & 1 & 1 & 1 & 1 \\
\hline $\mathrm{a}$ & Baccharis pteronioides DC. & & & 1 & & 1 \\
\hline $\mathrm{a}$ & Baccharis salicifolia (Ruiz y Pav.) Pers. & & & 1 & & 1 \\
\hline $\mathrm{a}$ & Cirsium sp nov. & & & 1 & & 1 \\
\hline $\mathrm{a}$ & Eupatorium areolare DC. & 1 & 1 & 1 & 1 & 1 \\
\hline $\mathrm{a}$ & Eupatorium cronquistii (King y H. Rob) B.L. Turner & 1 & 1 & & 1 & 1 \\
\hline A & Eupatorium sp. 1 & 1 & 1 & 1 & 1 & 1 \\
\hline $\mathrm{a}$ & Eupatorium sp.2 & & & 1 & & 1 \\
\hline hp & Piqueria triflora Hemsl. & 1 & 1 & & 1 & \\
\hline $\mathrm{a}$ & Podachaenium eminens (Lag.) Sch. Bip. & & 1 & 1 & 1 & 1 \\
\hline A & Senecio angulifolius DC. & 1 & & & 1 & \\
\hline A & Senecio salignus DC. & 1 & 1 & 1 & 1 & 1 \\
\hline hp & Stevia sp. & & 1 & & 1 & \\
\hline $\mathrm{a}$ & Vernonia capreifolia Gleason & & & 1 & & 1 \\
\hline ha & $\begin{array}{l}\text { Desconocida } \\
\text { Begoniaceae }\end{array}$ & & & 1 & & 1 \\
\hline hp & $\begin{array}{l}\text { Begonia calderonii Standl. } \\
\text { Betulaceae }\end{array}$ & 1 & & & 1 & 1 \\
\hline A & Carpinus tropicalis (J.D. Sm.) Lundell & 1 & 1 & 1 & 1 & 1 \\
\hline
\end{tabular}




\begin{tabular}{|c|c|c|c|c|c|c|}
\hline \multirow[t]{2}{*}{ FV } & \multirow[t]{2}{*}{ Familias y especies } & \multicolumn{3}{|c|}{$\begin{array}{c}\text { Tipo de } \\
\text { vegetación }\end{array}$} & \multicolumn{2}{|c|}{$\begin{array}{l}\text { Tipo de } \\
\text { borde }\end{array}$} \\
\hline & & BMM & BP & C & BB & BC \\
\hline A & Ostrya virginiana (Mill.) K. Koch & 1 & & & 1 & \\
\hline A & $\begin{array}{l}\text { Alnus acuminata Kunth subsp. arguta (Schltdl.) Furlow } \\
\text { Blechnaceae }\end{array}$ & 1 & 1 & & & 1 \\
\hline $\mathrm{hp}$ & Blechnum appendiculatum Willd. & 1 & & & 1 & \\
\hline hp & $\begin{array}{l}\text { Blechnum occidentale } \mathrm{L} . \\
\text { Boraginaceae }\end{array}$ & 1 & & & 1 & \\
\hline A & Tournefortia sp. & 1 & & & 1 & 1 \\
\hline $\mathrm{a}$ & $\begin{array}{l}\text { Tournefortia petiolaris A. DC. } \\
\text { Buddlejaceae }\end{array}$ & & & 1 & & 1 \\
\hline A & $\begin{array}{l}\text { Buddleja parviflora Kunth } \\
\text { Caprifoliaceae }\end{array}$ & 1 & & 1 & & 1 \\
\hline A & $\begin{array}{l}\text { Viburnum hartwegii Benth. } \\
\text { Caryophyllaceae }\end{array}$ & 1 & 1 & & 1 & \\
\hline ha & $\begin{array}{l}\text { Arenaria lanuginosa (Michx.) Rohrb. } \\
\text { Celastraceae }\end{array}$ & 1 & 1 & & 1 & \\
\hline $\mathrm{L}$ & Celastraceae & 1 & & & 1 & \\
\hline $\mathrm{L}$ & Celastrus pringlei Rose & 1 & & & 1 & \\
\hline A & Perrottetia longistylis Rose & 1 & & & 1 & 1 \\
\hline A & $\begin{array}{l}\text { Zinowiewia concinna Lundell } \\
\text { Clethraceae }\end{array}$ & 1 & 1 & 1 & 1 & 1 \\
\hline A & $\begin{array}{l}\text { Clethra fragrans L.M. González y R. Ramírez } \\
\text { Cornaceae }\end{array}$ & 1 & 1 & 1 & 1 & 1 \\
\hline A & $\begin{array}{l}\text { Cornus disciflora Moc. y Sessé ex DC. } \\
\text { Cyperaceae }\end{array}$ & 1 & 1 & 1 & 1 & 1 \\
\hline $\mathrm{h}$ & $\begin{array}{l}\text { Rhynchospora sp. } \\
\text { Dennstaedtiaceae }\end{array}$ & & 1 & 1 & 1 & 1 \\
\hline hp & $\begin{array}{l}\text { Pteridium arachnoideum (Kaulf.) Maxon } \\
\text { Ericaceae }\end{array}$ & 1 & 1 & & 1 & 1 \\
\hline A & $\begin{array}{l}\text { Arbutus xalapensis Kunth } \\
\text { Euphorbiaceae }\end{array}$ & & 1 & & 1 & 1 \\
\hline $\mathrm{a}$ & Chiropetalum schiedeanum (Muell. Arg.) Pax & 1 & 1 & 1 & 1 & 1 \\
\hline $\mathrm{a}$ & $\begin{array}{l}\text { Euphorbia schlechtendalii Boiss. var. pacifica McVaugh } \\
\text { Fabaceae }\end{array}$ & 1 & 1 & 1 & 1 & 1 \\
\hline a & Crotalaria longirostrata Hook. y Arn. & & 1 & 1 & 1 & \\
\hline $\mathrm{a}$ & Desmodium cf. sumichrastii (Schindl.) Standl. & 1 & & 1 & 1 & 1 \\
\hline $\mathrm{a}$ & Desmodium sp. & & 1 & & 1 & \\
\hline ha & $\begin{array}{l}\text { Phaseolus coccineus L. } \\
\text { Fagaceae }\end{array}$ & 1 & & & 1 & \\
\hline A & Quercus candicans Née & 1 & 1 & & 1 & \\
\hline A & Quercus salicifolia Liebm. & 1 & 1 & 1 & 1 & 1 \\
\hline A & Quercus scytophylla Liebm. & & 1 & 1 & 1 & 1 \\
\hline A & Quercus uxoris McVaugh & 1 & 1 & & 1 & \\
\hline A & Quercus xalapensis Humb. y Bonpl. & 1 & 1 & 1 & 1 & 1 \\
\hline A & $\begin{array}{l}\text { Quercus sp. } \\
\text { Flacourtiaceae }\end{array}$ & & 1 & & 1 & \\
\hline A & Hasseltiopsis dioica (Benth.) Sleumer & 1 & & 1 & & 1 \\
\hline A & $\begin{array}{l}\text { Xylosma flexuosa (Kunth) Hemsl. } \\
\text { Geraniaceae }\end{array}$ & 1 & & 1 & 1 & 1 \\
\hline hp & Geranium seemannii Peyr. & 1 & & 1 & 1 & 1 \\
\hline
\end{tabular}




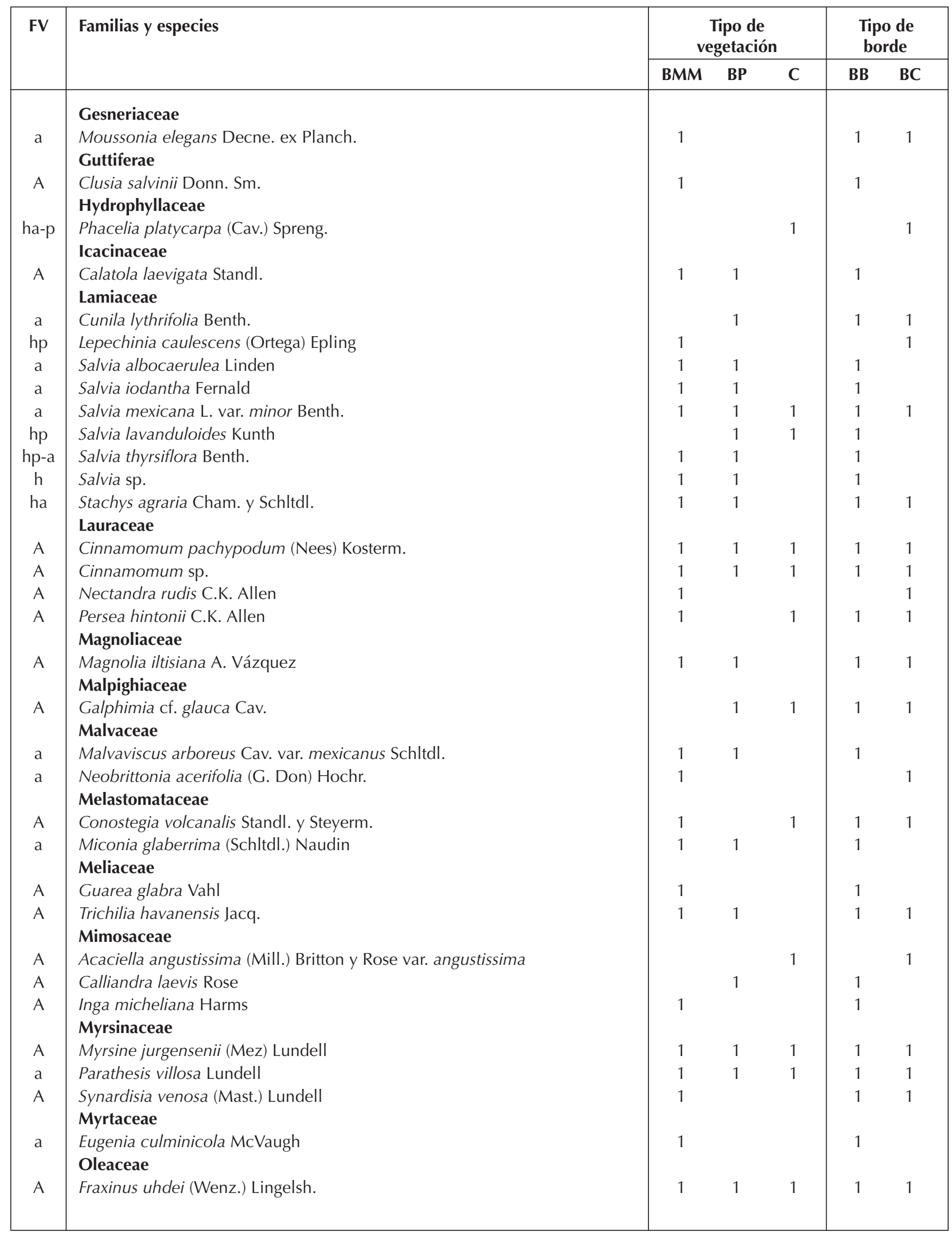




\begin{tabular}{|c|c|c|c|c|c|c|}
\hline \multirow[t]{2}{*}{ FV } & \multirow[t]{2}{*}{ Familias y especies } & \multicolumn{3}{|c|}{$\begin{array}{c}\text { Tipo de } \\
\text { vegetación }\end{array}$} & \multicolumn{2}{|c|}{$\begin{array}{l}\text { Tipo de } \\
\text { borde }\end{array}$} \\
\hline & & BMM & BP & C & BB & BC \\
\hline & Onagraceae & & & & & \\
\hline $\mathrm{a}$ & Fuchsia arborescens Sims & 1 & 1 & 1 & 1 & 1 \\
\hline $\mathrm{a}$ & Fuchsia encliandra Steud. subsp. encliandra & 1 & 1 & 1 & 1 & 1 \\
\hline $\mathrm{a}$ & $\begin{array}{l}\text { Fuchsia sp. } \\
\text { Piperaceae }\end{array}$ & 1 & & & 1 & \\
\hline $\mathrm{a}$ & $\begin{array}{l}\text { Piper pseudolindenii C. DC. } \\
\text { Pinaceae }\end{array}$ & 1 & & & 1 & \\
\hline A & $\begin{array}{l}\text { Pinus douglasiana Martínez } \\
\text { Poaceae }\end{array}$ & 1 & 1 & 1 & 1 & 1 \\
\hline $\mathrm{a}$ & Chusquea nelsonii Scribn. y J.G. Sm. & 1 & 1 & 1 & 1 & \\
\hline ha & Oplismenus burmanii (Retz.) Beauv. var. burmannii & 1 & 1 & & 1 & \\
\hline hp & Oplismenus compositum (L.) P. Beauv. & 1 & 1 & 1 & 1 & 1 \\
\hline hp & Panicum albomaculatum Scribn. & & 1 & & 1 & \\
\hline hp & Zea diploperennis H.H. Iltis, Doebley y R. Guzmán & & & 1 & & 1 \\
\hline hp & $\begin{array}{l}\text { Zeugites americana Willd. var.mexicana (Kunth) McVaugh } \\
\text { Polypodiaceae }\end{array}$ & 1 & 1 & 1 & 1 & 1 \\
\hline hp & Campyloneurum amphostenon (Kuntze ex Klotzsch) Fée & 1 & & & 1 & \\
\hline hp-e & Pecluma alfredii (Rosenst.) M.G. Price & 1 & & & 1 & \\
\hline hp-e & Pecluma cupreolepis (Evans) M.G. Price & 1 & & & 1 & \\
\hline hp-e & Phlebodium areolatum (Humb. y Bonpl. ex Willd.) J. Sm. & & 1 & & 1 & \\
\hline hp-e & Pleopeltis angusta Humb.y Bonpl. ex. Willd. var. angusta & 1 & & & 1 & \\
\hline hp & $\begin{array}{l}\text { Polypodium sp. } \\
\text { Ranunculaceae }\end{array}$ & 1 & & & 1 & \\
\hline hp & $\begin{array}{l}\text { Ranunculus petiolaris H.B.K. ex Don var. petiolaris } \\
\text { Rhamnaceae }\end{array}$ & 1 & 1 & & 1 & \\
\hline A & $\begin{array}{l}\text { Rhamnus hintonii M.C.Johnst. y L.A.Johnst. } \\
\text { Rosaceae }\end{array}$ & 1 & & & 1 & 1 \\
\hline A & Crataegus pubescens (Kunth) Steud. & & & 1 & & 1 \\
\hline A & Prunus cortapico Kerber ex Koehne & 1 & & 1 & & 1 \\
\hline A & Prunus persica (L.) Batsch. & & & 1 & & 1 \\
\hline $\mathrm{a}$ & $\begin{array}{l}\text { Rubus adenotrichos Schltdl. } \\
\text { Rubiaceae }\end{array}$ & & 1 & 1 & 1 & 1 \\
\hline $\mathrm{a}$ & Arachnothrys manantlanensis (Lorence) Borhidi & 1 & & & 1 & \\
\hline $\mathrm{a}$ & Chiococca pachyphylla Wernham & 1 & & & 1 & \\
\hline $\mathrm{a}$ & Hoffmania cuneatissima B. L. Rob. & 1 & 1 & & 1 & 1 \\
\hline $\mathrm{a}$ & $\begin{array}{l}\text { Rubiaceae } \\
\text { Sabiaceae }\end{array}$ & 1 & 1 & 1 & 1 & 1 \\
\hline A & Meliosma dentata (Liebm.) Urb. & 1 & 1 & & 1 & 1 \\
\hline A & $\begin{array}{l}\text { Meliosma nesites I.M. Johnst. } \\
\text { Scrophulariaceae }\end{array}$ & 1 & 1 & & 1 & \\
\hline $\mathrm{hp}$ & Castilleja arvensis Cham. y Schltdl. & & & 1 & 1 & \\
\hline hp & $\begin{array}{l}\text { Sibthorpia repens (Mutis ex L. f.) Kuntze } \\
\text { Solanaceae }\end{array}$ & 1 & & & & 1 \\
\hline $\mathrm{a}$ & Cestrum aurantiacum Lindl. & & 1 & & 1 & \\
\hline A & Cestrum nitidum M.Martens y Galeotti & 1 & 1 & & 1 & \\
\hline A & Cestrum terminale Francey & & & 1 & & 1 \\
\hline A & Cestrum sp.1 & & & 1 & & 1 \\
\hline $\mathrm{a}$ & Cestrum sp.2 & 1 & & & & 1 \\
\hline $\mathrm{a}$ & Lycianthes surotatensis Gentry & 1 & & & & 1 \\
\hline
\end{tabular}




\begin{tabular}{|c|c|c|c|c|c|c|}
\hline \multirow[t]{2}{*}{ FV } & \multirow[t]{2}{*}{ Familias y especies } & \multicolumn{3}{|c|}{$\begin{array}{l}\text { Tipo de } \\
\text { vegetación }\end{array}$} & \multicolumn{2}{|c|}{$\begin{array}{l}\text { Tipo de } \\
\text { borde }\end{array}$} \\
\hline & & BMM & BP & $\mathbf{C}$ & BB & BC \\
\hline a & Solanum aphyodendron S. Knapp & 1 & & & & 1 \\
\hline $\mathrm{a}$ & Solanum sp. 1 & 1 & 1 & 1 & 1 & 1 \\
\hline $\mathrm{a}$ & Solanum sp. 2 & 1 & 1 & 1 & 1 & 1 \\
\hline $\mathrm{a}$ & Solanum sp. 3 & 1 & 1 & 1 & 1 & 1 \\
\hline $\mathrm{a}$ & $\begin{array}{l}\text { Solanum nigricans M. Martens y Galeotti } \\
\text { Smilacaceae }\end{array}$ & 1 & & 1 & & 1 \\
\hline $\mathrm{L}$ & Smilax spinosa Mill. & 1 & & & & 1 \\
\hline $\mathrm{L}$ & $\begin{array}{l}\text { Smilax sp. } \\
\text { Styracaceae }\end{array}$ & 1 & & 1 & & 1 \\
\hline A & $\begin{array}{l}\text { Styrax argenteus C. Presl. } \\
\text { Symplocaceae }\end{array}$ & 1 & 1 & 1 & 1 & 1 \\
\hline A & $\begin{array}{l}\text { Symplocos citrea Lex. } \\
\text { Theaceae }\end{array}$ & 1 & 1 & 1 & 1 & 1 \\
\hline A & Cleyera integrifolia (Benth.) Choisy & 1 & & & 1 & \\
\hline A & Symplococarpon purpusii (Brandegee) Kobuski & 1 & 1 & & 1 & 1 \\
\hline A & $\begin{array}{l}\text { Ternstroemia dentisepala B. Bartholomew. } \\
\text { Thelypteridaceae }\end{array}$ & 1 & 1 & & 1 & \\
\hline hp & $\begin{array}{l}\text { Thelypteris sp. } \\
\text { Tiliaceae }\end{array}$ & 1 & & & & 1 \\
\hline A & Tilia americana L. var. mexicana (Schltdl.) Hardin & 1 & 1 & & 1 & 1 \\
\hline $\mathrm{a}$ & Triumfetta barbosa Lay & 1 & & & & 1 \\
\hline a & Triumfetta sp. 1 & & & 1 & & 1 \\
\hline a & $\begin{array}{l}\text { Triumfetta sp. } 2 \\
\text { Verbenaceae }\end{array}$ & 1 & & & & 1 \\
\hline A & Citharexylum mocinnii D. Don & 1 & 1 & 1 & 1 & 1 \\
\hline A & $\begin{array}{l}\text { Lippia umbellata Cav. } \\
\text { Violaceae }\end{array}$ & 1 & 1 & & 1 & \\
\hline hp & $\begin{array}{l}\text { Hybanthus elatus (Turcz.) Morton. } \\
\text { No identificadas }\end{array}$ & 1 & & & & 1 \\
\hline $\mathrm{L}$ & Bejucos & & & 1 & & 1 \\
\hline $\mathrm{h}$ & Hierbas & 3 & 3 & & 6 & \\
\hline $\mathrm{a}$ & Arbustos & & & 3 & & 3 \\
\hline \multicolumn{2}{|c|}{ Total de Familias } & 57 & 44 & 35 & 55 & 50 \\
\hline \multicolumn{7}{|c|}{ Especies por forma de vida } \\
\hline & - Árboles & 51 & 37 & 30 & 51 & 44 \\
\hline & - Arbustos & 33 & 25 & 27 & 32 & 36 \\
\hline & - Hierbas & 38 & 21 & 11 & 40 & 20 \\
\hline & - Bejucos & 4 & 1 & 3 & 3 & 4 \\
\hline \multicolumn{2}{|c|}{ Total de especies } & 126 & 84 & 71 & 126 & 104 \\
\hline
\end{tabular}

